QUARTERLY OF APPLIED MATHEMATICS

VOLUME LXIV, NUMBER 1

MARCH 2006, PAGES 61-91

S 0033-569X(06)00994-7

Article electronically published on January 24, 2006

\title{
WHEN IS A LINEAR SYSTEM CONSERVATIVE?
}

\author{
BY \\ JARMO MALINEN (Institute of Mathematics, P.O. Box 1100, Helsinki University of Technology, \\ FIN-02015 HUT, Finland), \\ OLOF J. STAFFAnS (Department of Mathematics Åbo Akademi University, FIN-20500 Åbo, \\ Finland), \\ AND \\ GEORGE WEISS (Department of Electric and Electronic Engineering, Imperial College London, \\ Exhibition Road, London SW7 2BT, United Kingdom)
}

\begin{abstract}
We consider infinite-dimensional linear systems without a-priori wellposedness assumptions, in a framework based on the works of M. Livšic, M. S. Brodskiü, Y. L. Smuljan, and others. We define the energy in the system as the norm of the state squared (other, possibly indefinite quadratic forms will also be considered). We derive a number of equivalent conditions for a linear system to be energy preserving and hence, in particular, well posed. Similarly, we derive equivalent conditions for a system to be conservative, which means that both the system and its dual are energy preserving. For systems whose control operator is one-to-one and whose observation operator has dense range, the equivalent conditions for being conservative become simpler, and reduce to three algebraic equations.
\end{abstract}

1. Introduction. In this paper we give a number of algebraic characterizations of energy preserving and of conservative linear systems in terms of the operators appearing in a differential/algebraic state space description of the system. Such algebraic characterizations may be useful for recognizing that certain linear partial differential or delay equations describe energy preserving or conservative systems. Our conditions do not include an a-priori well-posedness assumption, but well-posedness follows if the system is energy preserving.

Received January 6, 2005.

2000 Mathematics Subject Classification. Primary 47A48, 47N70, 93B28, 93C25.

Key words and phrases. Conservative system, energy preserving system, well-posed linear system, regular linear system, operator node, Cayley transform.

This research was supported by grants from the European Research Network on Systems Identification (ERNSI), the Academy of Finland under grant 203991, the Mittag-Leffler Institute (Sweden) and EPSRC (UK), under the Portfolio Partnership grant GR/S61256/01.

E-mail address: Jarmo.Malinen@hut.fi

E-mail address: Olof.Staffans@abo.fi

E-mail address: G.Weiss@imperial.ac.uk 
To make our paper easier to understand, first we give finite-dimensional versions of our results. Let the system $\Sigma$ be described by

$$
\left\{\begin{array}{l}
\dot{x}(t)=A x(t)+B u(t), \\
y(t)=C x(t)+D u(t),
\end{array}\right.
$$

where $A: X \rightarrow X, B: U \rightarrow X, C: X \rightarrow Y$, and $D: U \rightarrow Y$ are operators (representable by matrices) between the finite-dimensional spaces $U, X$, and $Y$. The differential equation in (1.1) has, for any $u \in C([0, \infty) ; U)$ and any $x_{0} \in X$, a unique solution $x \in C^{1}([0, \infty) ; X)$ with $x(0)=x_{0}$. We call $u(t), x(t)$, and $y(t)$ the input, state, and output of the system at time $t \geq 0$.

The system $\Sigma$ is energy preserving if the balance equation

$$
\|x(T)\|^{2}+\int_{0}^{T}\|y(t)\|^{2} \mathrm{~d} t=\|x(0)\|^{2}+\int_{0}^{T}\|u(t)\|^{2} \mathrm{~d} t
$$

holds for any input signal $u$, any initial state $x(0)$, and any time $T \geq 0$. This corresponds to interpreting $\|x(T)\|^{2}$ as the energy stored in the system at time $T$. Examples of such systems occur often in physics and engineering; for example, they could be mechanical systems without friction or circuits built from capacitors, inductors, and transformers, with a suitable choice of inputs and outputs.

The dual system of $\Sigma$, denoted $\Sigma^{d}$, is described by

$$
\left\{\begin{array}{l}
\dot{z}(t)=A^{*} z(t)+C^{*} w(t), \\
v(t)=B^{*} z(t)+D^{*} u(t),
\end{array}\right.
$$

where $w(t), z(t)$, and $v(t)$ are the input, state, and output of $\Sigma^{d}$ at time $t \geq 0$.

Proposition 1.1. The above system $\Sigma$ is energy preserving if and only if

$$
A+A^{*}=-C^{*} C, \quad B^{*}=-D^{*} C, \quad D^{*} D=I .
$$

The dual system $\Sigma^{d}$ is energy preserving if and only if

$$
A+A^{*}=-B B^{*}, \quad C=-D B^{*}, \quad D D^{*}=I .
$$

Proof. The balance equation (1.2) can be written in differential form:

$$
\frac{\mathrm{d}}{\mathrm{d} t}\|x(t)\|^{2}=\|u(t)\|^{2}-\|y(t)\|^{2} .
$$

We can write both sides of this equality as quadratic functions in $x(t)$ and $u(t)$, and the corresponding terms must be equal. By a straightforward computation, (1.4) is equivalent to (1.6). This argument can be found in, e.g., Ball [4.

By a similar argument, (1.5) is equivalent to the dual version of (1.6), which concerns the dual system $\Sigma^{d}$ described by (1.3).

We call the first equations in (1.4) and in (1.5) Lyapunov equations. This is not the standard terminology; normally, equations like $P A+A^{*} P=-C^{*} C$, in the unknown $P$, are called Lyapunov equations. Here we have $P=I$, but later we shall encounter $P \neq I$, when we allow a weighting matrix in the definition of the energy. Similarly, we call the second equations in (1.4) and in (1.5) cross-term equations, and the third equations (concerning $D^{*} D$ and $D D^{*}$ ) indicator equations. 
If both $\Sigma$ and $\Sigma^{d}$ are energy preserving, then $\Sigma$ is called conservative. Finite-dimensional energy preserving systems $\Sigma$ which satisfy Ker $D^{*}=\{0\}$ are actually conservative, as the following proposition shows.

Proposition 1.2. For the finite-dimensional system $\Sigma$ described by (1.1), the following statements are equivalent:

(i) $\Sigma$ is conservative.

(ii) $\operatorname{Ker} D^{*}=\{0\}$ and (1.4) holds.

(iii) Ker $D=\{0\}$ and (1.5) holds.

Proof. It follows from the previous proposition that if $\Sigma$ is conservative, then both (1.4) and (1.5) hold. In particular, $D$ is unitary, so that Ker $D=\{0\}$ and Ker $D^{*}=\{0\}$. Thus, statement (i) implies (ii) and (iii).

Now suppose that statement (ii) holds. Then it is easy to see that $D$ is unitary. From $B^{*}=-D^{*} C$ and $D D^{*}=I$ we get $C=-D B^{*}$. This, together with $D^{*} D=I$ implies $C^{*} C=B B^{*}$, so that (1.5) holds. Now (1.4) and (1.5) together imply (i), according to Proposition 1.1. The fact that (iii) implies (i) is proved similarly.

REMARK 1.3. The conditions (ii) and (iii) can be reformulated as follows:

(ii') $\operatorname{dim} U=\operatorname{dim} Y$ and (1.4) holds.

(iii') $\operatorname{dim} U=\operatorname{dim} Y$ and (1.5) holds.

Indeed, if $\operatorname{dim} U=\operatorname{dim} Y$, then $D^{*} D=I$ is equivalent to $D D^{*}=I$, and this implies that Ker $D=\{0\}$ and Ker $D^{*}=\{0\}$. The converse direction is also easy.

For a system $\Sigma$ of the form (1.1), the matrix-valued function

$$
\mathbf{G}(s)=C(s-A)^{-1} B+D, \quad s \in \rho(A),
$$

is called its transfer function. It follows from (1.4) that if $\Sigma$ is energy preserving, then $\mathbf{G}$ is inner, i.e., it is analytic on the open right half-plane $\mathbb{C}_{+}$and

$$
\mathbf{G}^{*}(i \omega) \mathbf{G}(i \omega)=I, \quad \omega \in \mathbb{R} .
$$

It follows from (1.5) that if the dual system $\Sigma^{d}$ is energy preserving, then we have $\mathbf{G}(i \omega) \mathbf{G}(i \omega)^{*}=I$ for all $\omega \in \mathbb{R}$, so that $\mathbf{G}$ is co-inner.

Proposition 1.2 gives the simplest characterization of conservative systems in finite dimensions, via three algebraic equations (either (1.4) or (1.5)) and a null-space condition. Unfortunately, this proposition cannot be generalized to infinite-dimensional systems, for several reasons. First, $\operatorname{dim} U=\operatorname{dim} Y$ is not necessarily true for conservative systems with an infinite-dimensional state space; see [33, Section 6]. Second, regardless if the condition $\operatorname{dim} U=\operatorname{dim} Y$ holds, the operator $D$ need not have a clear meaning in infinite dimensions. For certain systems, called regular systems, there is a natural candidate for $D$, the limit of the transfer function at $+\infty$. However, if $D$ is obtained in this way for a conservative system, it need not satisfy $D^{*} D=I$ or $D D^{*}=I$ (for example, for a delay line $D=0$, see [34, Section 7]). Third, the transfer function of an infinite-dimensional energy preserving system need not be inner; it can even be zero (see [33, Section 6]).

The aim of this paper is to give a characterization of conservativity which is amenable to infinite-dimensional generalization, with possibly unbounded $A, B$, and $C$. In order to state the finite-dimensional version of this characterization in a simple way, we make 
the following additional assumptions:

$$
\text { Ker } B=\{0\}, \quad \text { Ker } C^{*}=\{0\} .
$$

We call conservative systems satisfying (1.7) tory systems 1 In some sense, tory systems have no "redundant" or "wasted" subspaces in $U$ and $Y$, so that all the information is circulated through the state space. Tory systems may seem a rather special subclass of conservative systems, but in fact they are, in a sense, universal. Indeed, in Section 4 we prove that for any conservative system, the input and output spaces can be decomposed into two orthogonal components, $U=U_{1} \oplus U_{0}$ and $Y=Y_{1} \oplus Y_{0}$, with the following property: The system can be decomposed into a tory system acting from the input space $U_{1}$ to the output space $Y_{1}$ and a unitary constant acting from $U_{0}$ to $Y_{0}$. In most PDE examples of conservative systems (see [29, [33, 34]), this unitary constant is absent, and the system is tory.

Proposition 1.4. Let $\Sigma$ be a finite-dimensional system described by (1.1) and satisfying (1.7). Then $\Sigma$ is conservative if and only if

$$
A+A^{*}=-C^{*} C, \quad A+A^{*}=-B B^{*}, \quad C=-D B^{*} .
$$

Note that one of the equations in (1.8) is taken from (1.4) and two are taken from (1.5). The last equation in (1.8) could be replaced by the corresponding equation $B^{*}=-D^{*} C$ in (1.4), leading to an equivalent set of three equations.

Proof. The necessity of (1.8) follows from Proposition 1.1. Conversely, suppose that (1.8) is satisfied. If we substitute $C=-D B^{*}$ and $C^{*}=-B D^{*}$ into the first equation in (1.8) and compare the result with the second equation, we obtain that $B B^{*}=B D^{*} D B^{*}$. Since Ker $B=\{0\}$, it follows that $B^{*}=D^{*} D B^{*}$, or equivalently, $B=B D^{*} D$. Using again that Ker $B=\{0\}$, we obtain $D^{*} D=I$. Applying $D^{*}$ to both sides of $C=-D B^{*}$, we obtain $B^{*}=-D^{*} C$, so that all the equations in (1.4) hold. According to Proposition 1.1. $\Sigma$ is energy preserving.

Substituting $B^{*}=-D^{*} C$ into the second equation in (1.8) and comparing the result with the first equation, we get $C^{*} D D^{*} C=C^{*} C$. This implies, using twice $\operatorname{Ker} C^{*}=\{0\}$, that $D D^{*}=I$. Thus, all the equations in (1.5) hold. According to Proposition 1.1, $\Sigma^{d}$ is energy preserving, so that $\Sigma$ is conservative.

Propositions 1.1 and 1.4 presented earlier can easily be generalized to a restrictive class of infinite-dimensional systems, called state linear systems in the book of Curtain and Zwart [10]. The input, state, and output spaces of such systems, denoted $U, X$, and $Y$, are Hilbert spaces. Any state linear system is described by (1.1), where $A$ generates a strongly continuous semigroup on $X$, while $B: U \rightarrow X, C: X \rightarrow Y$, and $D: U \rightarrow Y$ are bounded. The dual of the system described by (1.1) is the system described by (1.3). In this class, energy preserving and conservative systems are defined similarly as for finite-dimensional systems. For state linear systems, the Lyapunov equation in (1.4) should be understood as an equation on $\mathcal{D}(A)$, and the dual Lyapunov equation in (1.5) should be understood as an equation on $\mathcal{D}\left(A^{*}\right)$. If the system is conservative, then it

\footnotetext{
${ }^{1}$ The Conservative party in the UK is also known as the Tory party.
} 
follows from the two Lyapunov equations and the boundedness of $B$ and $C$ that, in fact, $\mathcal{D}(A)=\mathcal{D}\left(A^{*}\right)$.

Proposition 1.5. Propositions 1.1 and 1.4 remain valid (without change) for state linear systems. Proposition 1.2 remains valid for systems with $\mathcal{D}(A)=\mathcal{D}\left(A^{*}\right)$.

The proofs are similar to those for finite-dimensional systems and we omit them.

Remark 1.6. Proposition 1.2 cannot be generalized to state linear systems without the extra assumption stated in the above proposition. Indeed, consider the following counterexample: Let $X=L^{2}[0, \infty), U=Y=\mathbb{C}$,

$$
(A x)(\xi)=\frac{\mathrm{d} x(\xi)}{\mathrm{d} \xi}, \quad \mathcal{D}(A)=H_{0}^{1}(0, \infty)
$$

(this is the generator of the right shift semigroup). Then $\mathcal{D}\left(A^{*}\right)=H^{1}(0, \infty)$ and $A+A^{*}=$ 0 on $\mathcal{D}(A)$. Take $B=0, C=0$, and $D=1$. Then (1.4) holds, so that (by the last proposition) the system determined by $A, B, C, D$ is energy preserving. We have Ker $D^{*}=\{0\}$, but clearly this system is not conservative.

In Sections 3-4 we will give results analogous to Propositions 1.1 and 1.4 in a much more general framework than the state linear systems considered in Proposition 1.5. Our framework permits us to consider systems with unbounded control and observation operators, in particular, linear PDE systems with boundary control and/or boundary observation, as well as certain delay equations.

We shall state in this introduction a slightly simplified version of the result that we consider to be the most important. For this we have to make some assumptions and introduce some concepts. Consider the system $\Sigma$ described by

$$
\left\{\begin{array}{l}
\dot{x}(t)=A x(t)+B u(t) \\
y(t)=\bar{C} x(t)+D u(t)
\end{array}\right.
$$

similar to (1.1). Here, $A$ generates a strongly continuous semigroup on the state space $X$, a Hilbert space. We define $X_{1}=\mathcal{D}(A)$, with the norm given by $\|x\|_{X_{1}}=\|(\alpha-A) x\|_{X}$, where $\alpha \in \rho(A)$. We define $X_{-1}$ to be the completion of $X$ with respect to the norm $\|x\|_{X_{-1}}=\left\|(\alpha-A)^{-1} x\right\|_{X}$ (for more details on these spaces, see Section 2). We assume that $B \in \mathcal{L}\left(U ; X_{-1}\right)$, and we introduce the space $Z$ by

$$
Z=X_{1}+(\alpha-A)^{-1} B U
$$

where $\alpha \in \rho(A)$. Here, $A$ really stands for the extension of $A$ to an operator in $\mathcal{L}\left(X ; X_{-1}\right)$, so that $(\alpha-A)^{-1} \in \mathcal{L}\left(X_{-1} ; X\right)$. This space is sometimes called the solution space. $Z$ is a Hilbert space with the norm given by

$$
\|z\|_{Z}^{2}=\inf \left\{\|x\|_{X_{1}}^{2}+\|v\|_{U}^{2} \mid x \in X_{1}, v \in U, z=x+(\alpha-A)^{-1} B v\right\} .
$$

If we change $\alpha \in \rho(A)$ in the last two formulas, then the space $Z$ remains the same, while its norm changes to an equivalent one. We have $X_{1} \subset Z \subset X$, with continuous embeddings, and $(\alpha-A)^{-1} B \in \mathcal{L}(U ; Z)$ for all $\alpha \in \rho(A)$. We assume that in (1.9), $\bar{C} \in \mathcal{L}(Z ; Y)$ and $D \in \mathcal{L}(U ; Y)$.

The assumptions made so far (on $A, B, \bar{C}$, and $D$ ) do not imply that (1.9) defines a well-posed system in the sense of Salamon [16, 17, Smuljan [18, Staffans [19, 24] 
or Weiss 31 (see also Definition 2.7). However, they do guarantee the existence of state trajectories $x$ and output functions $y$ for sufficiently smooth input functions $u$ and compatible initial states $x_{0}$, as stated below:

Proposition 1.7. With the above assumptions, take $T>0$ and let $u \in C^{2}([0, T] ; U)$ and $x_{0} \in X$ satisfy the compatibility condition $A x_{0}+B u(0) \in X$. Then the first equation in (1.9) has a unique classical solution $x \in C^{1}([0, T] ; X)$ satisfying the initial condition $x(0)=x_{0}$. Moreover, we have $x \in C([0, T] ; Z)$, so that $y \in C([0, T] ; Y)$ can be defined by the second equation in (1.9).

This is a consequence of Proposition [2.6 in the next section.

In the sequel we will use the notation $\left[\begin{array}{l}X \\ Y\end{array}\right]$ for $X \times Y$. Based on the above proposition, we define for each $T>0$ the space

$$
\mathcal{V}_{T}=\left\{\left[\begin{array}{c}
x_{0} \\
u
\end{array}\right] \in\left[\begin{array}{c}
X \\
C^{2}([0, T] ; U)
\end{array}\right] \mid A x_{0}+B u(0) \in X\right\}
$$

for which the solution of (1.9) is well defined on [0,T], and the operator $\Sigma_{T}: \mathcal{V}_{T} \rightarrow$ $\left[\begin{array}{c}X \\ C([0, T] ; Y)\end{array}\right]$ which associates to $\left[\begin{array}{c}x_{0} \\ u\end{array}\right]$ the corresponding pair $\left[\begin{array}{c}x(T) \\ y\end{array}\right]$. Thus,

$$
\left[\begin{array}{c}
x(T) \\
y
\end{array}\right]=\Sigma_{T}\left[\begin{array}{c}
x_{0} \\
u
\end{array}\right] .
$$

$\Sigma_{T}$ can be regarded as a densely defined operator from $\left[\begin{array}{c}X \\ L^{2}([0, T] ; U)\end{array}\right]$ to $\left[\begin{array}{c}X \\ L^{2}([0, T] ; Y)\end{array}\right]$.

We define the operator $C$ to be the restriction of $\bar{C}$ to $X_{1}$, so that $C \in \mathcal{L}\left(X_{1} ; Y\right)$. Let $X_{-1}^{d}$ be the completion of $X$ with respect to the norm $\|x\|_{X_{-1}^{d}}=\left\|\left(\bar{\alpha}-A^{*}\right)^{-1} x\right\|_{X}$ where $\alpha \in \rho(A)$. This space is the dual of $X_{1}$ with respect to the pivot space $X$, so that $C^{*} \in \mathcal{L}\left(Y ; X_{-1}^{d}\right)$ (see Section 2 for more details).

Definition 1.8. Let $\Sigma$ be the system determined by $A, B, \bar{C}, D$ satisfying the assumptions listed after (1.9). We call $\Sigma$ energy preserving if $\Sigma_{T}$ is isometric for all $T>0$, i.e., (1.2) holds. In this case $\Sigma_{T}$ has a unique extension to an isometric operator from $\left[\begin{array}{c}X \\ L^{2}([0, T] ; U)\end{array}\right]$ to $\left[\begin{array}{c}X \\ L^{2}([0, T] ; Y)\end{array}\right]$, denoted by the same symbol. We call $\Sigma$ conservative if it is isometric and the operators $\Sigma_{T}$ (after the extension described above) are unitary. We call $\Sigma$ tory if it is conservative and (1.7) holds.

Later we shall introduce a generalization of the concept of a conservative system (defined using the dual system) which allows the use of (possibly indefinite) weighting operators on $U, X$, and $Y$.

At last, we are now able to state a simplified version of our main result:

Theorem 1.9. Consider the system $\Sigma$ determined by $A, B, \bar{C}, D$ as in (1.9), and assume that (1.7) holds. Then the following conditions are equivalent:

(i) $\Sigma$ is conservative (hence, tory);

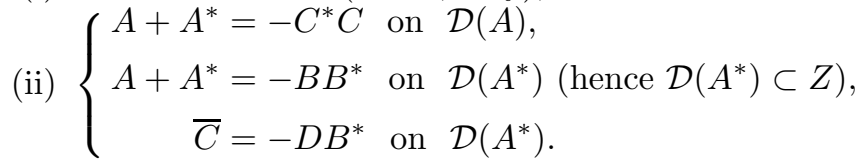


This is a consequence of Theorem 4.4. Note that in the first equation of (ii), $A^{*}$ is actually extended to an operator from $X$ to $X_{-1}^{d}$, while in the second equation of (ii), $A$ is extended to an operator from $X$ to $X_{-1}$.

Now we outline how weighting operators on the spaces $U, X$, and $Y$ can be introduced. Our discussion here concerns finite-dimensional systems only, but the infinite-dimensional case will be treated in the later sections. The energy balance equation (1.6) can be replaced by the more general equation

$$
\frac{\mathrm{d}}{\mathrm{d} t}\langle x(t), P x(t)\rangle=\langle u(t), R u(t)\rangle-\langle y(t), J y(t)\rangle,
$$

with $P=P^{*} \in \mathcal{L}(X), J=J^{*} \in \mathcal{L}(Y)$, and $R=R^{*} \in \mathcal{L}(U)$. A system $\Sigma$ defined as in (1.1) which satisfies this balance equation is called $(R, P, J)$-energy preserving. It can be shown that $\Sigma$ is $(R, P, J)$-energy preserving if and only if

$$
P A+A^{*} P=-C^{*} J C, \quad B^{*} P=-D^{*} J C, \quad R=D^{*} J D .
$$

We formally get these equations from (1.4) by replacing the adjoint operators $A^{*}, B^{*}$, $C^{*}$, and $D^{*}$ in (1.4) by the corresponding adjoints $A^{\dagger}=P^{-1} A^{*} P, B^{\dagger}=R^{-1} B^{*} P$, $C^{\dagger}=P^{-1} C^{*} J$, and $D^{\dagger}=R^{-1} D^{*} J$ computed with respect to the possibly indefinite inner products induced by $R, P$, and $J$, e.g., $\langle A x, P z\rangle=\left\langle x, P A^{\dagger} z\right\rangle$.

For the remainder of this discussion (in this section) we assume that $R, P$, and $J$ are invertible. We say that the system $\Sigma$ is $(R, P, J)$-conservative if it is $(R, P, J)$-energy preserving and its dual system $\Sigma^{d}$ (see (1.3) $)$ is $\left(J^{-1}, P^{-1}, R^{-1}\right)$-energy preserving. The fact that $\Sigma^{d}$ is $\left(J^{-1}, P^{-1}, R^{-1}\right)$-energy preserving is equivalent to the dual version of (1.14), namely:

$$
P^{-1} A^{*}+A P^{-1}=-B R^{-1} B^{*}, \quad C P^{-1}=-D R^{-1} B^{*}, \quad J^{-1}=D R^{-1} D^{*} .
$$

Note that (1.14) and (1.15) are equivalent whenever $D$ is invertible. Conversely, (1.14) and (1.15) imply that $D$ is invertible. Hence, if $D$ is invertible, then $\Sigma$ is $(R, P, J)$-energy preserving if and only if it is $(R, P, J)$-conservative. If $R, P$, and $J$ are positive and invertible, then it is possible to renorm the spaces $U, X$, and $Y$, and to reduce this result to the case where $R=I, P=I$, and $J=I$.

We complete this section with a glance at previous work. Early work on conservative systems dates back to Brodskiır [6], 7], Helton [11, Livšic [13, Livšic and Yantsevich [14, Sz.-Nagy and Foiaş 27, and Tsekanovski and Smuljan [28, to mention only a few. Most of these references consider discrete-time systems, where the difficulties are considerably smaller. Our system theory framework is analogous to what is called an operator colligation, or an operator node, or a Livšic-Brodskiu node in the works mentioned above for Hilbert space contractions (in the discrete-time case) or for contraction semigroups (in the continuous-time case). The discrete time version of our tory systems are known under the name Julia colligations; see [1].

The continuous-time analogue of an operator colligation was first formally introduced by Smuljan [18] in the Soviet school, but it can be found implicitly in some other works 
from the same time, such as Salamon [16. The approach by Smuljan is influenced by the scattering theory of Lax and Phillips [12. Arov and Nudelman [3] use the Cayley transform (of systems) to convert earlier known discrete-time results to Smuljan's (and our) continuous-time setting. The same transform is also used in [15].

For other background material on conservative systems in the above sense, we refer to Arov [2, Ball [4], Staffans [21, [22, 23], Staffans and Weiss [26], Tucsnak and Weiss [29, 34, Weiss [32] and Weiss, Staffans and Tucsnak 33]. A comprehensive reference to many aspects of linear infinite-dimensional systems is Staffans [24].

We mention that our results here can be applied to the class of systems considered in [29, 32], 34] (which are described by second-order differential equations in Hilbert spaces). The fact that these systems are conservative was proved in 34] by a detailed argument, which could be replaced by an application of Theorem 1.9.

2. Background on linear systems. In this section, we introduce a class of linear infinite-dimensional systems, called system nodes. System nodes are not necessarily well posed (in the usual sense, which will be recalled), but they have well-defined state trajectories and output functions corresponding to smooth input functions and compatible initial states, as we show in Proposition 2.6. We also introduce the dual of a system node.

In the theory of well-posed linear systems (see 24, 25], 31]) we often encounter a strongly continuous semigroup $\mathbb{T}$ acting on a Hilbert space $X$, with generator $A$, which determine two additional Hilbert spaces, denoted $X_{1}$ and $X_{-1}$. We have already seen them in Theorem [1.9, and here we recall their main properties. In fact, we work in a slightly more general framework, allowing $A$ to be an unbounded operator but not necessarily a semigroup generator.

Proposition 2.1. Let $X$ be a Hilbert space and let $A: \mathcal{D}(A) \subset X \rightarrow X$ be a closed, densely defined linear operator with a nonempty resolvent set $\rho(A)$. Take $\alpha \in \rho(A)$.

(i) For each $x \in \mathcal{D}(A)$, define $\|x\|_{X_{1}}=\|(\alpha-A) x\|_{X}$. Then $\|\cdot\|_{X_{1}}$ is a norm on $X_{1}$ which makes $X_{1}$ into a Hilbert space, and $A \in \mathcal{L}\left(X_{1} ; X\right)$. The operator $(\alpha-A)^{-1}$ maps $X$ isometrically onto $X_{1}$.

(ii) Let $X_{-1}$ be the completion of $X$ with respect to the norm $\|x\|_{X_{-1}}=$ $\left\|(\alpha-A)^{-1} x\right\|_{X}$. Then $X$ is a Hilbert space and $A$ has a unique extension to an operator $A_{-1} \in \mathcal{L}\left(X ; X_{-1}\right)$. The operator $\left(\alpha-A_{-1}\right)^{-1}$ maps $X_{-1}$ isometrically onto $X$. Moreover, $A_{-1}$ and $A$ are unitarily similar:

$$
A_{-1}=\left(\alpha-A_{-1}\right) A\left(\alpha-A_{-1}\right)^{-1} .
$$

(iii) If $A$ is the generator of a strongly continuous semigroup $\mathbb{T}=\left(\mathbb{T}^{t}\right)_{t \geq 0}$ on $X$, then the restriction $\mathbb{T}_{1}=\mathbb{T}_{\mid X_{1}}$ of $\mathbb{T}$ to $X_{1}$ is a strongly continuous semigroup on $X_{1}$. $\mathbb{T}$ is unitarily similar to $\mathbb{T}_{1}$, since

$$
\mathbb{T}^{t}=(\alpha-A) \mathbb{T}_{1}^{t}(\alpha-A)^{-1} \quad \forall t \geq 0 .
$$

The generator of $\mathbb{T}_{1}$ is $A_{1}$, the restriction of $A$ to $\mathcal{D}\left(A^{2}\right)$. 
(iv) If the semigroup $\mathbb{T}$ is as in (iii), then it has a unique extension to a strongly continuous semigroup $\mathbb{T}_{-1}$ on $X_{-1}$ which is unitarily similar to $\mathbb{T}$, since

$$
\mathbb{T}_{-1}^{t}=\left(\alpha-A_{-1}\right) \mathbb{T}^{t}\left(\alpha-A_{-1}\right)^{-1} \quad \forall t \geq 0
$$

The generator of $\mathbb{T}_{-1}$ is $A_{-1}$.

All of this is well known (and easy to prove) even in the Banach space context. The operator $A$ is often taken to be a semigroup generator also in parts (i) and (ii). For the proof and further details we refer to [30, Section 3] or [24, Section 3.6]. By iterating the construction in Proposition 2.1, we get an infinite sequence of Hilbert spaces ... $\subset$ $X_{2} \subset X_{1} \subset X \subset X_{-1} \subset X_{-2} \subset \ldots$ with continuous and dense embeddings, and the corresponding operators $A_{n}$ and semigroups $\mathbb{T}_{n}$.

We remark that the choice of $\alpha \in \rho(A)$ does not change the spaces $X_{1}$ or $X_{-1}$, since different values of $\alpha \in \rho(A)$ lead to equivalent norms in $X_{1}$ and $X_{-1}$.

In the sequel, we shall use the notation $\left[\begin{array}{l}X \\ Y\end{array}\right]$ for $X \times Y$.

Definition 2.2. Let $U, X$, and $Y$ be Hilbert spaces. An operator

$$
S:=\left[\begin{array}{l}
A \& B \\
C \& D
\end{array}\right]:\left[\begin{array}{l}
X \\
U
\end{array}\right] \supset \mathcal{D}(S) \rightarrow\left[\begin{array}{l}
X \\
Y
\end{array}\right]
$$

is called an operator node on $(U, X, Y)$ if it has the following structure:

(i) $A$ is a densely defined operator on $X$ with a nonempty resolvent set (which we extend to an operator $A_{-1} \in \mathcal{L}\left(X ; X_{-1}\right)$ as explained in Proposition 2.1).

(ii) $B \in \mathcal{L}\left(U ; X_{-1}\right)$.

(iii) $\mathcal{D}(S)=V$, where $V:=\left\{\left[\begin{array}{l}x \\ u\end{array}\right] \in\left[\begin{array}{l}X \\ U\end{array}\right] \mid A_{-1} x+B u \in X\right\}$.

(iv) $A \& B=\left[\begin{array}{ll}A_{-1} & B\end{array}\right]_{\mid V}$; we use on $V$ the graph norm of $A \& B$ :

$$
\left\|\left[\begin{array}{l}
x \\
u
\end{array}\right]\right\|_{V}^{2}:=\|x\|_{X}^{2}+\|u\|_{U}^{2}+\left\|A_{-1} x+B u\right\|_{X}^{2} .
$$

Then it clearly follows that $A \& B \in \mathcal{L}(V, X)$.

(v) $C \& D \in \mathcal{L}(V ; Y)$.

If, in addition to the above, $A$ generates a strongly continuous semigroup on $X$, then $S$ is called a system node.

Definition 2.2 is roughly analogous to what is known as an operator node or a colligation in the works of Livšic [13, Livšic and Yantsevich [14, Brodskiur [7, Sz.-Nagy and Foias [27, for Hilbert space contractions (in the discrete-time case) or for contraction semigroups (in the continuous-time case). Smuljan developed a theory of infinitedimensional systems in [18, which was later used by Arov and Nudelman [3]. His approach resembles the theory of well-posed linear systems in general, and its formulation in this section in particular. System nodes in the above framework have been used in Staffans 21], 22], 23].

REmark 2.3. Since $\left[\begin{array}{ll}A_{-1} & B\end{array}\right]$ is bounded from $[\underset{U}{X}]$ to $X_{-1}$, it follows that $A \& B$ is closed (as a densely defined operator from $\left[\begin{array}{l}X \\ U\end{array}\right]$ to $X$ ). This implies that $V$ is complete, hence a Hilbert space. It also implies that $S$ is closed (as a densely defined operator from $\left[\begin{array}{l}X \\ U\end{array}\right]$ to $\left.\left[\begin{array}{c}X \\ Y\end{array}\right]\right)$. Indeed, this follows from the facts that $A \& B$ is closed, that $C \& D$ has the same domain as $A \& B$, and that $C \& D$ is continuous with respect to the graph norm of $A \& B$. 
We call $A \in \mathcal{L}\left(X_{1} ; X\right)$ the main operator of $S, B \in \mathcal{L}\left(U ; X_{-1}\right)$ is its control operator, and $C \& D \in \mathcal{L}(V ; Y)$ is its combined observation/feedthrough operator. From the last operator we can extract $C \in \mathcal{L}\left(X_{1} ; Y\right)$, the observation operator of $S$, defined by

$$
C x:=C \& D\left[\begin{array}{l}
x \\
0
\end{array}\right], \quad x \in X_{1} .
$$

A short computation shows that for each $\alpha \in \rho(A)$, the operator

$$
E_{\alpha}:=\left[\begin{array}{cc}
I & \left(\alpha-A_{-1}\right)^{-1} B \\
0 & I
\end{array}\right]
$$

is a bounded bijection from $\left[\begin{array}{c}X \\ U\end{array}\right]$ onto itself and also from $\left[\begin{array}{c}X_{1} \\ U\end{array}\right]$ onto $V$. Since $\left[\begin{array}{c}X_{1} \\ U\end{array}\right]$ is dense in $\left[\begin{array}{c}X \\ U\end{array}\right]$, this implies that $V$ is also dense in $\left[\begin{array}{l}X \\ U\end{array}\right]$. Since the second column of $E_{\alpha}$ maps $U$ into $V$, we can define the transfer function of $S$ by

$$
\mathbf{G}(s):=C \& D\left[\begin{array}{c}
\left(s-A_{-1}\right)^{-1} B \\
I
\end{array}\right], \quad s \in \rho(A),
$$

which is an $\mathcal{L}(U ; Y)$-valued analytic function. Clearly, for any two $\alpha, \beta \in \rho(A)$,

$$
\mathbf{G}(\alpha)-\mathbf{G}(\beta)=C\left[\left(\alpha-A_{-1}\right)^{-1}-\left(\beta-A_{-1}\right)^{-1}\right] B .
$$

In our construction, the operator node $S$, the observation operator $C$, and the transfer function $\mathbf{G}$ are determined by the operators $A, B$, and $C \& D$. Alternatively, $S$ and $\mathbf{G}$ may be constructed from $A, B, C$, and the value of $\mathbf{G}$ at one point in $\rho(A)$. More precisely, given $A \in \mathcal{L}\left(X_{1} ; X\right)$ and $B \in \mathcal{L}\left(U ; X_{-1}\right)$ we first define $V$ and $A \& B$ as in Definition 2.2. Then, for any $C \in \mathcal{L}\left(X_{1} ; Y\right), E \in \mathcal{L}(U ; Y)$, and $\alpha \in \rho(A)$, there is a unique $C \& D \in \mathcal{L}(V ; Y)$ such that (2.1) holds and the function $\mathbf{G}$ from (2.3) satisfies $\mathbf{G}(\alpha)=E$. This operator is explicitly given by

$$
C \& D\left[\begin{array}{l}
x \\
u
\end{array}\right]:=C\left[x-\left(\alpha-A_{-1}\right)^{-1} B u\right]+E u .
$$

We will also need the (unbounded) adjoint of an operator node. Let $A^{*}$ be the unbounded adjoint of $A$, which is defined on $\mathcal{D}\left(A^{*}\right) \subset X$. In addition to the spaces $X_{1}$ and $X_{-1}$ induced by the operator $A$, we will also need the corresponding spaces induced by $A^{*}$. We denote the analogue of $X_{1}$ by $X_{1}^{d}$ and the analogue of $X_{-1}$ by $X_{-1}^{d}$, so that $X_{1}^{d} \subset X \subset X_{-1}^{d}$. The corresponding norms will be denoted by $\|\cdot\|_{X_{1}^{d}}$ and $\|\cdot\|_{X_{-1}^{d}}$. It is easy to check that $X_{-1}^{d}$ is the dual of $X_{1}$ with respect to the pivot space $X$. To explain this in greater detail, note that for any $x \in X$,

$$
\|x\|_{X_{-1}^{d}}=\sup _{z \in X_{1},\|z\|_{X_{1}} \leq 1}|\langle z, x\rangle| .
$$

This implies that the scalar product on $X$, restricted to $X_{1} \times X$, has a unique continuous extension to $X_{1} \times X_{-1}^{d}$ and (2.6) remains valid for $x \in X_{-1}^{d}$. Similarly, $X_{-1}$ is the dual of $X_{1}^{d}$ with respect to the pivot space $X$.

In the sequel we shall also need the extensions of $A^{*}$ and $\mathbb{T}^{*}$ to $X_{-1}^{d}\left(\right.$ with $\left.\mathcal{D}\left(A^{*}\right)=X\right)$ and also their restrictions to $X_{1}^{d}$. However, rather than introducing any further notation, 
we shall denote these extensions and restrictions by $A^{*}$ respectively $\mathbb{T}^{*}$. Likewise, in the sequel we write $A$ instead of $A_{n}$ and $\mathbb{T}$ instead of $\mathbb{T}_{n}$.

We will always identify $U, X$, and $Y$ with their duals.

Proposition 2.4. Let $S$ be an operator node on $(U, X, Y)$ with main operator $A$, control operator $B$, observation operator $C$, and transfer function $\mathbf{G}$. Then the (unbounded) adjoint of $S$ is given by

$$
S^{*}=\left[\begin{array}{l}
{[A \& B]^{d}} \\
{[C \& D]^{d}}
\end{array}\right]: V^{d} \rightarrow\left[\begin{array}{l}
X \\
U
\end{array}\right]
$$

with domain

$$
\mathcal{D}\left(S^{*}\right)=V^{d}:=\left\{\left[\begin{array}{l}
x \\
y
\end{array}\right] \in\left[\begin{array}{l}
X \\
Y
\end{array}\right] \mid A^{*} x+C^{*} y \in X\right\},
$$

where $[A \& B]^{d}=\left[\begin{array}{ll}A^{*} & C^{*}\end{array}\right]_{\mid V^{d}}$ and, for every $\alpha \in \rho\left(A^{*}\right)$,

$$
[C \& D]^{d}\left[\begin{array}{l}
x \\
y
\end{array}\right]:=B^{*}\left[x-\left(\bar{\alpha}-A^{*}\right)^{-1} C^{*} y\right]+\mathbf{G}(\alpha)^{*} y .
$$

Furthermore, $S^{*}$ is an operator node on $(Y, X, U)$ with main operator $A^{*}$, so that if $S$ is a system node, then so is $S^{*}$.

The operator $S^{*}$ is called the dual node of $S$. It is easy to see that the transfer functions of $S^{*}$ and $S$ are related by $\mathbf{G}^{d}(s)=\mathbf{G}(\bar{s})^{*}$, for all $s \in \rho\left(A^{*}\right)$.

Proof. Choose $\alpha \in \rho(A)$ and let $E_{\alpha} \in \mathcal{L}\left(\left[\begin{array}{c}X \\ U\end{array}\right]\right)$ be given by (2.2). Since $E_{\alpha}$ is invertible,

$$
E_{\alpha}^{*} S^{*}=\left(S E_{\alpha}\right)^{*} \text {. }
$$

A straightforward algebraic manipulation using (2.1) and (2.3) gives

$$
S E_{\alpha}=\left[\begin{array}{c}
A \& B \\
C \& D
\end{array}\right]\left[\begin{array}{cc}
I & (\alpha-A)^{-1} B \\
0 & I
\end{array}\right]=\left[\begin{array}{cc}
A & \alpha(\alpha-A)^{-1} B \\
C & \mathbf{G}(\alpha)
\end{array}\right] .
$$

The unbounded adjoint of $S E_{\alpha}$ is given by

$$
\left(S E_{\alpha}\right)^{*}\left[\begin{array}{l}
x \\
y
\end{array}\right]=\left[\begin{array}{cc}
A^{*} & C^{*} \\
B^{*} \bar{\alpha}\left(\bar{\alpha}-A^{*}\right)^{-1} & \mathbf{G}(\alpha)^{*}
\end{array}\right]\left[\begin{array}{l}
x \\
y
\end{array}\right], \quad\left[\begin{array}{l}
x \\
y
\end{array}\right] \in V^{d},
$$

where $V^{d}$ is the space defined in the statement of the proposition: Indeed, if we regard $S E_{\alpha}$ as a bounded operator form $\left[\begin{array}{c}X_{1} \\ U\end{array}\right]$ to $\left[\begin{array}{c}X \\ Y\end{array}\right]$, then its adjoint is clearly given by (2.9), mapping $\left[\begin{array}{c}X \\ Y\end{array}\right]$ into $\left[\begin{array}{c}X_{-1}^{d} \\ U\end{array}\right]$. The unbounded adjoint of $S E_{\alpha}$ must be the restriction of this bounded adjoint to its natural domain $V^{d}$ consisting of those $\left[\begin{array}{l}x \\ y\end{array}\right] \in\left[\begin{array}{c}X \\ Y\end{array}\right]$ for which $\left(S E_{\alpha}\right)^{*}\left[\begin{array}{l}x \\ y\end{array}\right] \in\left[\begin{array}{l}X \\ U\end{array}\right]$. From (2.7) and (2.9) we get for all $\left[\begin{array}{l}x \\ y\end{array}\right] \in V^{d}$ the following expressions for $[A \& B]^{d}\left[\begin{array}{l}x \\ y\end{array}\right]$ and $[C \& D]^{d}\left[\begin{array}{l}x \\ y\end{array}\right]$ :

$$
\begin{aligned}
S^{*}\left[\begin{array}{l}
x \\
y
\end{array}\right] & =E_{\alpha}^{-*}\left(S E_{\alpha}\right)^{*}\left[\begin{array}{l}
x \\
y
\end{array}\right] \\
& =\left[\begin{array}{cr}
I & 0 \\
-B^{*}\left(\bar{\alpha}-A^{*}\right)^{-1} & I
\end{array}\right]\left[\begin{array}{c}
A^{*} x+C^{*} y \\
B^{*} \bar{\alpha}\left(\bar{\alpha}-A^{*}\right)^{-1} x+\mathbf{G}(\alpha)^{*} y
\end{array}\right] \\
& =\left[\begin{array}{c}
A^{*} x+C^{*} y \\
B^{*}\left(x-\left(\bar{\alpha}-A^{*}\right)^{-1} C^{*} y\right)+\mathbf{G}(\alpha)^{*} y
\end{array}\right]=\left[\begin{array}{l}
{[A \& B]^{d}} \\
{[C \& D]^{d}}
\end{array}\right]\left[\begin{array}{l}
x \\
y
\end{array}\right] .
\end{aligned}
$$


Checking Definition 2.2 we find that $S^{*}$ is an operator node on $(Y, X, U)$ with $\mathcal{D}\left(S^{*}\right)=$ $V^{d}$ and main operator $A^{*}$. If $A$ is the generator of a strongly continuous semigroup, then so is $A^{*}$, hence $S^{*}$ is then a system node.

As the following proposition shows, every operator node induces a natural decomposition in its input and output spaces that separate the "nontrivial" part of the node from its trivial "static" part.

Proposition 2.5. Let $S:=\left[\begin{array}{c}A \& B \\ C \& D\end{array}\right]$ be an operator node on $(U, X, Y)$ with $\mathcal{D}(S)=V$, main operator $A$, control operator $B$, and observation operator $C$. Decompose the spaces $U$ and $Y$ into the (not necessarily orthogonal) direct sums of closed subspaces

$$
U=\left[\begin{array}{l}
U_{1} \\
U_{0}
\end{array}\right], \quad Y=\left[\begin{array}{l}
Y_{1} \\
Y_{0}
\end{array}\right], \quad U_{0}=\operatorname{Ker} B, \quad Y_{1}=\overline{\operatorname{Ran} C} .
$$

These decompositions induce a (unique) decomposition of $S$ into

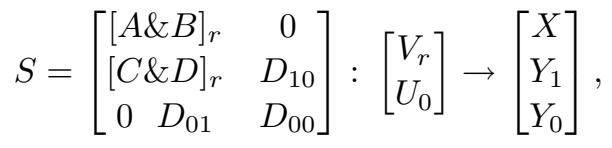

where $S_{r}:=\left[\begin{array}{l}{[A \& B]_{r}} \\ {[C \& D]_{r}}\end{array}\right]$ is an operator node on $\left(U_{1}, X, Y_{1}\right)$ with $\mathcal{D}\left(S_{r}\right)=V_{r}$. The main operator of $S_{r}$ is $A$, its control operator $B_{r}$ is obtained from $B=\left[\begin{array}{ll}B_{r} & 0\end{array}\right]$, and its observation operator $C_{r}$ is $C$, with range space $Y_{1} . B_{r}$ is one-to-one and $C_{r}$ has dense range, $D_{01} \in \mathcal{L}\left(U_{1} ; Y_{0}\right), D_{10} \in \mathcal{L}\left(U_{0} ; Y_{1}\right)$, and $D_{00} \in \mathcal{L}\left(U_{0} ; Y_{0}\right)$. The transfer function $\mathbf{G}$ of $S$ has the decomposition

$$
\mathbf{G}(s)=\left[\begin{array}{cc}
\mathbf{G}_{r}(s) & D_{10} \\
D_{01} & D_{00}
\end{array}\right], \quad s \in \rho(A),
$$

where $\mathbf{G}_{r}$ is the transfer function of $S_{r}$. We have $\left[\begin{array}{c}x \\ u_{1} \\ u_{0}\end{array}\right] \in V$ if and only if $\left[\begin{array}{c}x \\ u_{1}\end{array}\right] \in V_{r}$.

Proof. Let $\left[\begin{array}{l}x \\ u\end{array}\right] \in V$. We split $u$ into $u=\left[\begin{array}{l}u_{1} \\ u_{0}\end{array}\right] \in\left[\begin{array}{l}U_{1} \\ U_{0}\end{array}\right]$. Since $B\left[\begin{array}{l}u_{1} \\ u_{0}\end{array}\right]=\left[\begin{array}{ll}B_{r} & 0\end{array}\right]\left[\begin{array}{l}u_{1} \\ u_{0}\end{array}\right]=$ $B_{r} u_{1}$ for all $\left[\begin{array}{l}u_{1} \\ u_{0}\end{array}\right] \in\left[\begin{array}{l}U_{1} \\ U_{0}\end{array}\right]$, we have $B\left[\begin{array}{c}0 \\ u_{0}\end{array}\right]=0$. This implies that $\left[\begin{array}{c}0 \\ u_{0}\end{array}\right] \in V$, hence also $\left[\begin{array}{c}x \\ u_{1}\end{array}\right] \in V$. In other words, $V=\left[\begin{array}{l}V_{r} \\ U_{0}\end{array}\right]$, where $V_{r}=\left\{\left[\begin{array}{l}x \\ u\end{array}\right] \in V \mid u \in U_{1}\right\}$. We split $S$ accordingly into $S=\left[\begin{array}{ll}S_{r} & S_{0}\end{array}\right]$. These operators can be regarded as the restrictions of the original (unbounded) operator $S$ to the subspaces $\left[\begin{array}{c}X \\ U_{1}\end{array}\right]$ respectively $\left[\begin{array}{c}0 \\ U_{0}\end{array}\right]$ of $\left[\begin{array}{c}X \\ U\end{array}\right]$. Both of these are closed, and this implies that both $S_{r}$ and $S_{0}$ are closed.

The domain of $S_{0}$ is $U_{0}$ and its range is contained in $\left[\begin{array}{l}0 \\ Y\end{array}\right]$, so by the closed graph theorem, $S_{0}=\left[\begin{array}{c}0 \\ D_{0}\end{array}\right]$ for some $D_{0} \in \mathcal{L}\left(U_{0} ; Y\right)$. Using the decomposition $Y=\left[\begin{array}{c}Y_{1} \\ Y_{0}\end{array}\right]$, we get $D_{0}=\left[\begin{array}{c}D_{10} \\ D_{00}\end{array}\right]$ and $S_{0}=\left[\begin{array}{c}0 \\ D_{10} \\ D_{00}\end{array}\right]$ where $D_{10} \in \mathcal{L}\left(U_{0} ; Y_{1}\right)$ and $D_{00} \in \mathcal{L}\left(U_{0} ; Y_{0}\right)$.

It is easy to see that $S_{r}$ is an operator node on $\left(U_{1}, X, Y_{1}\right)$ with domain $V_{r}$. Thus, if we decompose $S_{r}$ as $S_{r}=\left[\begin{array}{l}{[A \& B]_{r}} \\ {[C \& D]_{r}} \\ {[C \& D]_{0}}\end{array}\right]$ in accordance with the decomposition of its range space $\left[\begin{array}{c}X \\ Y_{1} \\ Y_{0}\end{array}\right]$, then $[C \& D]_{r} \in \mathcal{L}\left(V_{r} ; Y_{1}\right),[C \& D]_{0} \in \mathcal{L}\left(V_{r} ; Y_{0}\right)$, and both $S_{r}=\left[\begin{array}{l}{[A \& B]_{r}} \\ {[C \& D]_{r}}\end{array}\right]$ and $\left[\begin{array}{l}{[A \& B]_{r}} \\ {[C \& D]_{0}}\end{array}\right]$ are operator nodes, with domain $V_{r}$ and output space $Y_{1}$, respectively $Y_{0}$. We get the observation operators of these operator nodes by decomposing $C=\left[\begin{array}{l}C_{r} \\ C_{0}\end{array}\right]$. Since 
$Y_{1}=\overline{\operatorname{Ran} C}$, we have $\overline{\operatorname{Ran} C_{r}}=Y_{1}$ and $C_{0}=0$. The latter fact implies that $[C \& D]_{0}$ has a (unique) extension to an operator $\left[\begin{array}{ll}0 & D_{01}\end{array}\right] \in \mathcal{L}\left(\left[\begin{array}{l}X \\ U_{1}\end{array}\right] ; Y_{0}\right)$.

The given decomposition of the transfer function $\mathbf{G}$ is an immediate consequence of the decompositions of $B$ into $\left[\begin{array}{ll}B_{r} & 0\end{array}\right]$ and of $C \& D$ into $\left[\begin{array}{ccc}{[C \& D]_{r}} & D_{10} \\ 0 & D_{01} & D_{00}\end{array}\right]$.

Up to now, we have only treated $S$ as a closed unbounded operator to which we have applied certain algebraic manipulations, and we have said nothing about dynamical systems. If $S$ is a system node, i.e., if its main operator generates a strongly continuous semigroup, then $S$ defines a linear dynamical system of a rather general type (and this is the reason for calling $S$ a system node).

Proposition 2.6. Let $S=\left[\begin{array}{c}A \& B \\ C \& D\end{array}\right]$ be a system node on $(U, X, Y)$ with domain $V$. Let $u \in C^{2}([0, \infty) ; U)$ and $\left[\begin{array}{c}x_{0} \\ u(0)\end{array}\right] \in V$. Then the equation

$$
\left[\begin{array}{l}
\dot{x}(t) \\
y(t)
\end{array}\right]=S\left[\begin{array}{l}
x(t) \\
u(t)
\end{array}\right], \quad t \geq 0, \quad x(0)=x_{0}
$$

has a unique (classical) solution $\left[\begin{array}{l}x \\ y\end{array}\right]$ satisfying $x \in C^{1}([0, \infty) ; X) \cap C^{2}\left([0, \infty) ; X_{-1}\right)$, $\left[\begin{array}{l}x \\ u\end{array}\right] \in C([0, \infty) ; V)$, and $y \in C([0, \infty) ; Y)$.

Proof. Denote the main operator of $S$ by $A$, the semigroup generated by $A$ by $\mathbb{T}$, and the control operator of $S$ by $B$. Since $A \& B=\left[\begin{array}{ll}A & B\end{array}\right]_{\mid V}$, we can write the top half of (2.12) as an equation in $X_{-1}$ (here $A$ stands for $A_{-1}$, as agreed):

$$
\dot{x}(t)=A x(t)+B u(t), \quad t \geq 0, \quad x(0)=x_{0} .
$$

For each $u \in C([0, \infty) ; U)$ and $x_{0} \in X_{-1}$, the equation (2.13) has the unique mild solution (where $\mathbb{T}$ stands for $\mathbb{T}_{-1}$, as agreed):

$$
x(t):=\mathbb{T}^{t} x_{0}+\int_{0}^{t} \mathbb{T}^{t-\sigma} B u(\sigma) d \sigma, \quad t \geq 0 .
$$

We denote by $C_{0}([0, \infty) ; U)$ the space of continuous $U$-valued functions vanishing at infinity, and $C_{0}^{k}([0, \infty) ; U) \subset C_{0}([0, \infty) ; U)$ for $k=0,1,2$ consists of those functions whose $k$ first derivatives are also in $C_{0}([0, \infty) ; U)$. These are Banach spaces with the norms $\|u\|_{C_{0}^{k}([0, \infty) ; U)}:=\max _{0 \leq j \leq k, t \geq 0}\left\|u^{(j)}(t)\right\|_{U}$. As the restriction of $x$ (as given in (2.14) ) to any finite interval $\left[0, t_{1}\right]$ depends only on $x_{0}$ and the restriction of $u$ to the same interval, it will be enough to prove the proposition for $u \in C_{0}^{2}([0, \infty) ; U$ ) (by redefining $u$ on $\left(t_{1}, \infty\right)$ if necessary).

We denote the (strongly continuous) backward shift semigroup on $C_{0}([0, \infty) ; U)$ by $\tau$, i.e., $\left(\tau^{t} u\right)(\theta)=u(\theta+t)$ for $\theta, t \geq 0$. The generator of $\tau$ is the differentiation operator, with domain $C_{0}^{1}([0, \infty) ; U)$. If we denote

$$
\mathfrak{B}^{t} u:=\int_{0}^{t} \mathbb{T}^{t-\sigma} B u(\sigma) d \sigma,
$$

then $\mathfrak{B}^{t} \in \mathcal{L}\left(C_{0}([0, \infty) ; U) ; X_{-1}\right)$ for each $t \geq 0$, and (2.14) can be written in the form $x(t)=\mathbb{T}^{t} x_{0}+\mathfrak{B}^{t} u, t \geq 0$. Introduce the Banach space $\mathbf{X}=\left[\begin{array}{c}X_{-1} \\ C_{0}([0, \infty) ; U)\end{array}\right]$, and for each 
$t \geq 0$, define the operator $\mathbf{T}^{t}: \mathbf{X} \rightarrow \mathbf{X}$ by

$$
\mathbf{T}^{t}:=\left[\begin{array}{cc}
\mathbb{T}^{t} & \mathfrak{B}^{t} \\
0 & \tau^{t}
\end{array}\right]
$$

A straightforward algebraic computation shows that $\mathbf{T}$ is a semigroup on $\mathbf{X}$, and it is also easy to see that $\mathbf{T}$ is strongly continuous. Moreover, for each $t \geq 0, \mathbf{T}^{t}\left[\begin{array}{c}x_{0} \\ u\end{array}\right]=\left[\begin{array}{c}x(t) \\ \tau^{t} u\end{array}\right]$, where $x$ is the mild solution in (2.14).

It is not difficult to verify that the generator of $\mathbf{T}$ is

$$
\mathbf{A}\left[\begin{array}{c}
x_{0} \\
u
\end{array}\right]=\left[\begin{array}{c}
A x_{0}+B u(0) \\
\dot{u}
\end{array}\right], \quad\left[\begin{array}{c}
x_{0} \\
u
\end{array}\right] \in \mathcal{D}(\mathbf{A})=\left[\begin{array}{c}
X \\
C_{0}^{1}([0, \infty) ; U)
\end{array}\right],
$$

and that the domain of $\mathbf{A}^{2}$ is

$$
\begin{aligned}
\mathcal{D}\left(\mathbf{A}^{2}\right) & =\left\{\left[\begin{array}{c}
x_{0} \\
u
\end{array}\right] \in \mathcal{D}(\mathbf{A}) \mid \mathbf{A}\left[\begin{array}{c}
x_{0} \\
u
\end{array}\right] \in \mathcal{D}(\mathbf{A})\right\} \\
& =\left\{\left[\begin{array}{c}
x_{0} \\
u
\end{array}\right] \in\left[\begin{array}{c}
X \\
C_{0}^{2}([0, \infty) ; U)
\end{array}\right] \mid\left[\begin{array}{c}
x_{0} \\
u(0)
\end{array}\right] \in V\right\} .
\end{aligned}
$$

Thus, the assumptions on the data in Proposition 2.6 have been chosen in such a way that $\left[\begin{array}{c}x_{0} \\ u\end{array}\right] \in \mathcal{D}\left(\mathbf{A}^{2}\right)$ (assuming that $u, u^{\prime}$, and $u^{\prime \prime}$ vanish at infinity).

By standard semigroup theory, if $\left[\begin{array}{c}x_{0} \\ u\end{array}\right] \in \mathcal{D}\left(\mathbf{A}^{2}\right)$, then $\mathbf{T}^{t}\left[\begin{array}{c}x_{0} \\ u\end{array}\right]=\left[\begin{array}{c}x(t) \\ \tau^{t} u\end{array}\right]$ is two times continuously differentiable in $\mathbf{X}$, so that $x \in C^{2}\left([0, \infty) ; X_{-1}\right)$. Since, from (2.13),

$$
x(t)=(\alpha-A)^{-1}[\alpha x(t)-\dot{x}(t)+B u(t)], \quad t \geq 0, \quad \alpha \in \rho(A),
$$

where $(\alpha-A)^{-1} \in \mathcal{L}\left(X_{-1} ; X\right)$ and $\alpha x-\dot{x}+B u \in C^{1}\left([0, \infty) ; X_{-1}\right)$, we obtain that $x \in C^{1}([0, \infty) ; X)$. From (2.15) it is easy to see that the operator $\Gamma$ defined by $\Gamma\left[\begin{array}{c}x_{0} \\ u\end{array}\right]=$ $\left[\begin{array}{c}x_{0} \\ u(0)\end{array}\right]$ is continuous from $\mathcal{D}\left(\mathbf{A}^{2}\right)$ to $V$. Since $\left[\begin{array}{l}x(t) \\ u(t)\end{array}\right]=\Gamma \mathbf{T}^{t}\left[\begin{array}{c}x_{0} \\ u\end{array}\right]$, it follows that $\left[\begin{array}{l}x \\ u\end{array}\right] \in$ $C([0, \infty) ; V)$, and this implies $y \in C([0, \infty) ; Y)$.

It is easy to show that under the assumptions of Proposition 2.6 if $\left[\begin{array}{c}x_{0} \\ u(0)\end{array}\right] \in V$ and $\ddot{u}(t)=O\left(e^{\omega t}\right)$ as $t \rightarrow \infty$ for some $\omega<\infty$, then the Laplace transforms $\hat{u}, \hat{x}$, and $\hat{y}$ satisfy

$$
\begin{aligned}
& \hat{x}(s)=(s-A)^{-1}\left[x_{0}+B \hat{u}(s)\right], \\
& \hat{y}(s)=C(s-A)^{-1} x_{0}+\mathbf{G}(s) \hat{u}(s),
\end{aligned}
$$

for all $s$ with sufficiently large real part (see, for example, [24, Lemma 4.7.11]).

We obtain the well-known class of well-posed linear systems by adding one more assumption to those in Definition 2.2

Definition 2.7. Let $S=\left[\begin{array}{c}A \& B \\ C \& D\end{array}\right]$ be a system node on $(U, X, Y)$. We call $S$ well posed if there is a function $K:(0, \infty) \rightarrow(0, \infty)$ such that for all $t>0$,

$$
\|x(t)\|_{X}^{2}+\|y\|_{L^{2}([0, t] ; Y)}^{2} \leq K(t)\left(\left\|x_{0}\right\|_{X}^{2}+\|u\|_{L^{2}([0, t] ; U)}^{2}\right),
$$

for all $x, y, x_{0}$, and $u$ satisfying the conditions of Proposition 2.6.

For a well-posed system node, the operator $\Sigma_{T}$ defined in (1.13) (on the domain $\mathcal{V}_{T}$ from $(1.12)$ ) can be extended to a bounded linear operator from $\left[\begin{array}{c}X \\ L^{2}([0, T] ; U)\end{array}\right]$ to $\left[\begin{array}{c}X \\ L^{2}([0, T] ; Y)\end{array}\right]$, denoted by the same symbol. The family $\left(\Sigma_{T}\right)_{T \geq 0}$ is then called a wellposed linear system in the terminology of [19, 25], 31], [33, and 34]. 
Definition 2.7 is easily seen to be equivalent to the definition used by Salamon in 16 . and by Smuljan in [18. (Salamon phrases it in terms of $A, B, C$, and $\mathbf{G}$ rather than in terms of the system node $S$.) Necessary and sufficient conditions for well-posedness were given in Curtain and Weiss 9]. If a system node is well posed, then its transfer function is bounded in some right half-plane. Conversely, every $\mathcal{L}(U ; Y)$-valued function which is analytic and bounded in some right half-plane can be interpreted as the transfer function of some well-posed system node: see, e.g., [17], 20], or [24] for details.

Definition 2.8. Let $S=\left[\begin{array}{l}A \& B \\ C \& D\end{array}\right]$ be an operator node on $(U, X, Y)$. We call $S$ compatible if its observation operator $C$ has an extension $\bar{C} \in \mathcal{L}(Z ; Y)$, where $Z$ is the Hilbert space in (1.10) and (1.11).

If $S$ is compatible and $\bar{C}$ is as above, then we can define $D \in \mathcal{L}(U ; Y)$ by

$$
D u=[C \& D]\left[\begin{array}{l}
x \\
u
\end{array}\right]-\bar{C} x, \quad\left[\begin{array}{l}
x \\
u
\end{array}\right] \in V .
$$

Indeed, it follows from the definition of $C$ in (2.1) that the above expression does not depend on $x$. Choosing $x=(s-A)^{-1} u$, we obtain

$$
D u=\mathbf{G}(s) u-\bar{C}(s-A)^{-1} B u, \quad s \in \rho(A),
$$

so $D$ is bounded (because $\mathbf{G}(s) \in \mathcal{L}(U ; Y)$ and $(s-A)^{-1} B$ is bounded from $U$ to $Z$ ). Thus, for any compatible operator node,

$$
\mathbf{G}(s)=D+\bar{C}(s-A)^{-1} B, \quad s \in \rho(A),
$$

which is similar to the usual formula for finite-dimensional systems. For a compatible operator node, the extension $\bar{C}$ is usually not unique. Every well-posed system node is compatible; this was shown in [25, Section 3]. For a compatible $S$, (2.12) can be written in the simpler form (1.9).

\section{Energy preserving systems.}

Definition 3.1. Let $U, X$, and $Y$ be Hilbert spaces, and let $R=R^{*} \in \mathcal{L}(U)$, $P=P^{*} \in \mathcal{L}(X)$, and $J=J^{*} \in \mathcal{L}(Y)$. We call a system node $S$ on $(U, X, Y)(R, P, J)$ energy preserving if for all $T>0$ we have

$$
\langle x(T), P x(T)\rangle_{X}+\int_{0}^{T}\langle y(t), J y(t)\rangle_{Y} d t=\left\langle x_{0}, P x_{0}\right\rangle_{X}+\int_{0}^{T}\langle u(t), R u(t)\rangle_{U} d t,
$$

for all $x, y, x_{0}$, and $u$ satisfying the conditions of Proposition 2.6. If $R=I, P=I$, and $J=I$, then we simply call the node energy preserving.

Note that (3.1) implies the well-posedness of $S$ if $P \geq \epsilon>0$ and $J \geq \epsilon>0$.

We present a number of characterizations of energy preserving system nodes.

TheOREM 3.2. Let $S=\left[\begin{array}{c}A \& B \\ C \& D\end{array}\right]$ be a system node on $(U, X, Y)$ with domain $V$, main operator $A$, control operator $B$, observation operator $C$, and transfer function $\mathbf{G}$. Let $S^{*}=\left[\begin{array}{l}{[A \& B]^{d}} \\ {[C \& D]^{d}}\end{array}\right]$ be the adjoint system node with domain $V^{d}$, and let $R=R^{*} \in \mathcal{L}(U)$, $P=P^{*} \in \mathcal{L}(X)$, and $J=J^{*} \in \mathcal{L}(Y)$. Then the following statements are equivalent:

(i) $S$ is $(R, P, J)$-energy preserving; 
(ii) for all $\left[\begin{array}{l}x_{0} \\ u_{0}\end{array}\right] \in V$,

$$
2 \Re\left\langle A \& B\left[\begin{array}{l}
x_{0} \\
u_{0}
\end{array}\right], P x_{0}\right\rangle_{X}=\left\langle u_{0}, R u_{0}\right\rangle_{U}-\left\langle C \& D\left[\begin{array}{l}
x_{0} \\
u_{0}
\end{array}\right], J[C \& D]\left[\begin{array}{l}
x_{0} \\
u_{0}
\end{array}\right]\right\rangle_{Y} ;
$$

(iii) the operator $\left[\begin{array}{ll}P & 0 \\ 0 & J\end{array}\right]\left[\begin{array}{cc}I & 0 \\ C \& D\end{array}\right]$ maps $V$ into $V^{d}$, and

$$
\left[\begin{array}{l}
{[A \& B]^{d}} \\
{[C \& D]^{d}}
\end{array}\right]\left[\begin{array}{cc}
P & 0 \\
0 & J
\end{array}\right]\left[\begin{array}{cc}
I & 0 \\
C \& D
\end{array}\right]=\left[\begin{array}{cc}
P & 0 \\
0 & R
\end{array}\right]\left[\begin{array}{cc}
-[A \& B] \\
0 & I
\end{array}\right] \quad \text { on } V \text {; }
$$

(iv) for some (hence, for all) $\alpha \in \rho(A)$, we have

$$
\begin{cases}P A+A^{*} P=-C^{*} J C & \text { on } X_{1}, \\ B^{*}\left(\bar{\alpha}-A^{*}\right)^{-1} P(\bar{\alpha}+A)+\mathbf{G}(\alpha)^{*} J C=0 & \text { on } X_{1}, \\ B^{*}\left(\bar{\alpha}-A^{*}\right)^{-1}(2 \Re \alpha) P(\alpha-A)^{-1} B+\mathbf{G}(\alpha)^{*} J \mathbf{G}(\alpha)=R & \text { on } U ;\end{cases}
$$

(v) for some (hence, for all) $\alpha \in \mathbb{C}_{+} \cap \rho(A)$, the operator

$$
\left[\begin{array}{ll}
\mathbf{A}(\alpha) & \mathbf{B}(\alpha) \\
\mathbf{C}(\alpha) & \mathbf{D}(\alpha)
\end{array}\right]=\left[\begin{array}{cc}
(\bar{\alpha}+A)(\alpha-A)^{-1} & \sqrt{2 \Re \alpha}(\alpha-A)^{-1} B \\
\sqrt{2 \Re \alpha} C(\alpha-A)^{-1} & \mathbf{G}(\alpha)
\end{array}\right],
$$

which is in $\mathcal{L}\left(\left[\begin{array}{c}X \\ U\end{array}\right] ;\left[\begin{array}{c}X \\ Y\end{array}\right]\right)$, is $\left(\left[\begin{array}{ll}P & 0 \\ 0 & R\end{array}\right],\left[\begin{array}{ll}P & 0 \\ 0 & J\end{array}\right]\right)$-isometric, i.e.,

$$
\left[\begin{array}{ll}
\mathbf{A}(\alpha) & \mathbf{B}(\alpha) \\
\mathbf{C}(\alpha) & \mathbf{D}(\alpha)
\end{array}\right]^{*}\left[\begin{array}{cc}
P & 0 \\
0 & J
\end{array}\right]\left[\begin{array}{ll}
\mathbf{A}(\alpha) & \mathbf{B}(\alpha) \\
\mathbf{C}(\alpha) & \mathbf{D}(\alpha)
\end{array}\right]=\left[\begin{array}{cc}
P & 0 \\
0 & R
\end{array}\right]
$$

In the case where $R=I, P=I$, and $R=I$, parts (i), (ii), and (v) of this theorem are closely related to [2, pp. 31-32] (more general weight operators are considered later in [2]). The operator $\left[\begin{array}{ll}\mathbf{A}(\alpha) & \mathbf{B}(\alpha) \\ \mathbf{C}(\alpha) & \mathbf{D}(\alpha)\end{array}\right] \in \mathcal{L}\left(\left[\begin{array}{c}X \\ U\end{array}\right] ;\left[\begin{array}{c}X \\ Y\end{array}\right]\right)$ defined in (v) is called the Cayley transform (with parameter $\alpha$ ) of $S$. Thus, a system node is $(R, P, J)$-energy preserving if and only if its Cayley transform is $\left(\left[\begin{array}{ll}P & 0 \\ 0 & R\end{array}\right],\left[\begin{array}{ll}P & 0 \\ 0 & J\end{array}\right]\right)$ - isometric.

Proof. (i) $\Rightarrow$ (ii): Let $\left[\begin{array}{l}x_{0} \\ u_{0}\end{array}\right] \in V$, let $u \in C^{2}([0, \infty) ; U)$ with $u(0)=u_{0}$, and let $x \in C^{1}([0, \infty) ; X)$ and $y \in C([0, \infty) ; Y)$ be as in Proposition 2.6. By differentiating (3.1) with respect to $T$, we get

$$
2 \Re\langle\dot{x}(t), P x(t)\rangle_{X}+\langle y(t), J y(t)\rangle_{Y}=\langle r(t), R u(t)\rangle_{U}, \quad t \geq 0,
$$

where $\dot{x}(t)=A \& B\left[\begin{array}{l}x(t) \\ u(t)\end{array}\right]$ and $y(t)=C \& D\left[\begin{array}{l}x(t) \\ u(t)\end{array}\right]$. Taking $t=0$ we get (3.2).

(ii) $\Rightarrow(\mathrm{i})$ : Let $\left[\begin{array}{l}x_{0} \\ u_{0}\end{array}\right] \in V$, let $u \in C^{2}([0, \infty) ; U)$ with $u(0)=u_{0}$, and let $x \in$ $C^{1}([0, \infty) ; X)$ and $y \in C([0, \infty) ; Y)$ be as in Proposition [2.6. Replace $\left[\begin{array}{l}x_{0} \\ u_{0}\end{array}\right]$ in (3.2) by $\left[\begin{array}{l}x(t) \\ u(t)\end{array}\right]$, observe that $A \& B\left[\begin{array}{l}x(t) \\ u(t)\end{array}\right]=\dot{x}(t)$, and integrate over $[0, T]$, to get (3.1).

(ii) $\Rightarrow$ (iii): By regrouping the terms in (3.2) we get the equivalent identity

$$
\begin{gathered}
\left\langle\left[\begin{array}{l}
A \& B \\
C \& D
\end{array}\right]\left[\begin{array}{l}
x_{0} \\
u_{0}
\end{array}\right],\left[\begin{array}{ll}
P & 0 \\
0 & J
\end{array}\right]\left[\begin{array}{cc}
I & 0 \\
C \& D
\end{array}\right]\left[\begin{array}{l}
x_{0} \\
u_{0}
\end{array}\right]\right\rangle_{\left[\begin{array}{l}
X \\
Y
\end{array}\right]} \\
=\left\langle\left[\begin{array}{l}
x_{0} \\
u_{0}
\end{array}\right],\left[\begin{array}{ll}
P & 0 \\
0 & R
\end{array}\right]\left[\begin{array}{cc}
-[A \& B \\
0 & I
\end{array}\right]\left[\begin{array}{l}
x_{0} \\
u_{0}
\end{array}\right]\right\rangle_{\left[\begin{array}{l}
X \\
Y
\end{array}\right]}
\end{gathered}
$$


valid for all $\left[\begin{array}{l}x_{0} \\ u_{0}\end{array}\right] \in V$. This, together with the polarization identity gives

$$
\begin{aligned}
\left\langle\left[\begin{array}{l}
A \& B \\
C \& D
\end{array}\right]\left[\begin{array}{l}
x_{1} \\
u_{1}
\end{array}\right],\left[\begin{array}{ll}
P & 0 \\
0 & J
\end{array}\right]\left[\begin{array}{cc}
I & 0 \\
C \& D
\end{array}\right]\left[\begin{array}{l}
x_{0} \\
u_{0}
\end{array}\right]\right\rangle_{\left[\begin{array}{l}
X \\
Y
\end{array}\right]} \\
=\left\langle\left[\begin{array}{l}
x_{1} \\
u_{1}
\end{array}\right],\left[\begin{array}{cc}
P & 0 \\
0 & R
\end{array}\right]\left[\begin{array}{cc}
-[A \& B \\
0 & I
\end{array}\right]\left[\begin{array}{l}
x_{0} \\
u_{0}
\end{array}\right]\right\rangle_{\left[\begin{array}{l}
X \\
Y
\end{array}\right]}
\end{aligned}
$$

for all $\left[\begin{array}{l}x_{0} \\ u_{0}\end{array}\right],\left[\begin{array}{l}x_{1} \\ u_{1}\end{array}\right] \in V$. For each fixed $\left[\begin{array}{l}x_{0} \\ u_{0}\end{array}\right] \in V$ the right-hand side above has an extension to a continuous functional on $\left[\begin{array}{c}X \\ Y\end{array}\right]$, so that $\left[\begin{array}{ll}P & 0 \\ 0 & R\end{array}\right]\left[\begin{array}{cc}I & 0 \\ C \& D\end{array}\right]\left[\begin{array}{l}x_{0} \\ u_{0}\end{array}\right] \in \mathcal{D}\left(S^{*}\right)=V^{d}$ and (3.3) holds (we have used Proposition 2.4).

(iii) $\Rightarrow$ (ii): This is the same computation done backwards.

(iii) $\Leftrightarrow$ (iv): We have seen that (3.3) is equivalent to (3.7). Let us replace $\left[\begin{array}{l}x_{0} \\ u_{0}\end{array}\right]$ and $\left[\begin{array}{l}x_{1} \\ u_{1}\end{array}\right]$ in (3.7) by $E_{\alpha}\left[\begin{array}{l}x_{2} \\ u_{2}\end{array}\right]$ respectively $E_{\alpha}\left[\begin{array}{l}x_{3} \\ u_{3}\end{array}\right]$, where $\left[\begin{array}{l}x_{2} \\ u_{2}\end{array}\right],\left[\begin{array}{l}x_{3} \\ u_{3}\end{array}\right] \in\left[\begin{array}{c}X_{1} \\ U\end{array}\right]$, and $E_{\alpha}$ is as in (2.2). Then (3.7) becomes (see (2.3) and (2.8))

$$
\begin{aligned}
{\left[\begin{array}{cc}
A & \alpha(\alpha-A)^{-1} B \\
C & \mathbf{G}(\alpha)
\end{array}\right]^{*} } & {\left[\begin{array}{ll}
P & 0 \\
0 & J
\end{array}\right]\left[\begin{array}{cc}
I & (\alpha-A)^{-1} B \\
C & \mathbf{G}(\alpha)
\end{array}\right] } \\
& =\left[\begin{array}{cc}
I & (\alpha-A)^{-1} B \\
0 & I
\end{array}\right]^{*}\left[\begin{array}{cc}
P & 0 \\
0 & R
\end{array}\right]\left[\begin{array}{cc}
-A & -\alpha(\alpha-A)^{-1} B \\
0 & I
\end{array}\right],
\end{aligned}
$$

or equivalently (see (2.9)),

$$
\begin{aligned}
{\left[\begin{array}{cc}
A^{*} & C^{*} \\
B^{*} \bar{\alpha}\left(\bar{\alpha}-A^{*}\right)^{-1} & \mathbf{G}(\alpha)^{*}
\end{array}\right]\left[\begin{array}{ll}
P & 0 \\
0 & J
\end{array}\right]\left[\begin{array}{cc}
I & (\alpha-A)^{-1} B \\
C & \mathbf{G}(\alpha)
\end{array}\right] } \\
=\left[\begin{array}{cc}
I & 0 \\
B^{*}\left(\bar{\alpha}-A^{*}\right)^{-1} & I
\end{array}\right]\left[\begin{array}{cc}
P & 0 \\
0 & R
\end{array}\right]\left[\begin{array}{cc}
-A & -\alpha(\alpha-A)^{-1} B \\
0 & I
\end{array}\right] .
\end{aligned}
$$

We may rewrite the above identity in the self-adjoint form

$$
\begin{gathered}
{\left[\begin{array}{cc}
P A+A^{*} P & \left(\alpha+A^{*}\right) P(\alpha-A)^{-1} B \\
B^{*}\left(\bar{\alpha}-A^{*}\right)^{-1} P(\bar{\alpha}+A) & B^{*}\left(\bar{\alpha}-A^{*}\right)^{-1}(2 \Re \alpha) P(\alpha-A)^{-1} B
\end{array}\right]} \\
+\left[\begin{array}{cc}
C^{*} J C & C^{*} J \mathbf{G}(\alpha) \\
\mathbf{G}(\alpha)^{*} J C & \mathbf{G}(\alpha)^{*} J \mathbf{G}(\alpha)
\end{array}\right]=\left[\begin{array}{ll}
0 & 0 \\
0 & R
\end{array}\right] .
\end{gathered}
$$

The top left corner of this identity is the first equation in (3.4). The top right corner is the identity $\left(\alpha+A^{*}\right) P(\alpha-A)^{-1} B+C^{*} J \mathbf{G}(\alpha)=0$ on $U$, which is the adjoint of the second equation in (3.4). The bottom left corner of (3.8) is the second equation in (3.4), and the bottom right corner is the third equation in (3.4).

(iv) $\Leftrightarrow$ (v): Recall that (3.4) is equivalent to (3.8). We get (3.6) by multiplying (3.8) by $\left[\begin{array}{cc}\sqrt{2 \Re \alpha}(\alpha-A)^{-1} & 0 \\ 0 & I\end{array}\right]$ to the right and by $\left[\begin{array}{cc}\sqrt{2 \Re \alpha}\left(\bar{\alpha}-A^{*}\right)^{-1} & 0 \\ 0 & I\end{array}\right]$ to the left.

REMARK 3.3. It follows from the proof of the equivalence of (iv) and (v) in Theorem 3.2 that each of the three equations in (3.4) is equivalent to the corresponding Cayley transformed equation (for all $\alpha \in \mathbb{C}_{+} \cap \rho(A)$ ):

$$
\left\{\begin{array}{l}
\mathbf{A}(\alpha)^{*} P \mathbf{A}(\alpha)+\mathbf{C}(\alpha)^{*} J \mathbf{C}(\alpha)=P, \\
\mathbf{B}(\alpha)^{*} P \mathbf{A}(\alpha)+\mathbf{D}(\alpha)^{*} J \mathbf{C}(\alpha)=0, \\
\mathbf{B}(\alpha)^{*} P \mathbf{B}(\alpha)+\mathbf{D}(\alpha)^{*} J \mathbf{D}(\alpha)=R .
\end{array}\right.
$$


We call the first equation (3.4) and the corresponding equation in (3.9) the (observation or primal) Lyapunov equation. We call the second equation the (primal) cross-term equation, and the last one we call the (primal) indicator equation. Similar terminology was introduced in Section 1, and the relationship between the corresponding equations is as follows: If $X$ is finite-dimensional and we take limits as $\alpha \rightarrow+\infty$ in (3.4), we obtain (1.4).

Note that the indicator equation in (3.4) implies the following: If $i \omega \in \rho(A)$ where $\omega \in \mathbb{R}$, then $\mathbf{G}(i \omega)^{*} J \mathbf{G}(i \omega)=R$.

Lemma 3.4. We use the notation of Theorem 3.2

(i) If the Lyapunov equation $P A+A^{*} P=-C^{*} J C$ holds on $X_{1}$, then the operator $Q(\alpha) \in \mathcal{L}\left(X_{1} ; Y\right)$ defined by

$$
Q(\alpha):=B^{*}\left(\bar{\alpha}-A^{*}\right)^{-1} P(\bar{\alpha}+A)+\mathbf{G}(\alpha)^{*} J C
$$

does not depend on $\alpha \in \rho(A)$.

(ii) If (i) holds and $Q(\alpha)=0$ for some (hence, for all) $\alpha \in \rho(A)$, then the operator $R(\beta, \gamma) \in \mathcal{L}(U)$ defined by

$$
R(\beta, \gamma):=B^{*}\left(\bar{\beta}-A^{*}\right)^{-1}(\bar{\beta}+\gamma) P(\gamma-A)^{-1} B+\mathbf{G}(\beta)^{*} J \mathbf{G}(\gamma)
$$

is self-adjoint and independent of $\beta, \gamma \in \rho(A)$.

Proof. (i) By using the Lyapunov equation $P A+A^{*} P=-C^{*} J C$ we can rewrite $Q(\alpha)$ in the form

$$
\begin{aligned}
Q(\alpha) & =B^{*}\left(\bar{\alpha}-A^{*}\right)^{-1}\left[\bar{\alpha} P-A^{*} P-C^{*} J C\right]+\mathbf{G}(\alpha)^{*} J C \\
& =B^{*}\left[P-\left(\bar{\alpha}-A^{*}\right)^{-1} C^{*} J C\right]+\mathbf{G}(\alpha)^{*} J C .
\end{aligned}
$$

If $\beta \in \rho(A)$, then by the dual version of (2.4)

$$
Q(\alpha)-Q(\beta)=\left\{B^{*}\left[\left(\bar{\beta}-A^{*}\right)^{-1}-\left(\bar{\alpha}-A^{*}\right)^{-1}\right] C^{*}+\mathbf{G}(\alpha)^{*}-\mathbf{G}(\beta)^{*}\right\} J C=0 .
$$

(ii) We first show that $R(\beta, \gamma)$ is independent of $\beta$. Let $\alpha, \beta, \gamma \in \rho(A)$. Since $Q(\gamma)=0$, we also have

$$
Q(\gamma)^{*}=\left(\gamma+A^{*}\right) P(\gamma-A)^{-1} B+C^{*} J \mathbf{G}(\gamma)=0 .
$$

By using this equation we can rewrite $R(\beta, \gamma)$ in the form

$$
\begin{aligned}
R(\beta, \gamma) & =B^{*}\left(\bar{\beta}-A^{*}\right)^{-1}\left[\left(\bar{\beta}-A^{*}\right) P(\gamma-A)^{-1} B-C^{*} J \mathbf{G}(\gamma)\right]+\mathbf{G}(\beta)^{*} J \mathbf{G}(\gamma) \\
& =B^{*}\left[P(\gamma-A)^{-1} B-\left(\bar{\beta}-A^{*}\right)^{-1} C^{*} J \mathbf{G}(\gamma)\right]+\mathbf{G}(\beta)^{*} J \mathbf{G}(\gamma) .
\end{aligned}
$$

Therefore, by the dual version of (2.4),

$$
\begin{aligned}
R(\alpha, \gamma)-R(\beta, \gamma)= & -B^{*}\left[\left(\bar{\alpha}-A^{*}\right)^{-1}-\left(\bar{\beta}-A^{*}\right)^{-1}\right] C^{*} J \mathbf{G}(\gamma) \\
& +\left[\mathbf{G}(\alpha)^{*}-\mathbf{G}(\beta)^{*}\right] J \mathbf{G}(\gamma)=0 .
\end{aligned}
$$

This shows that $R(\beta, \gamma)$ is independent of $\beta$. It must also be independent of $\gamma$ because of the fact that $R(\beta, \gamma)=R(\gamma, \beta)^{*}$, and $R(\gamma, \beta)$ does not depend on $\gamma$. Now, to see that $R(\beta, \gamma)$ is self-adjoint it suffices to take $\beta=\gamma$. 
The above lemma shows that the indicator equation (the last) in (3.4) may be replaced by the following equation, for some (hence for all) $\beta, \gamma \in \rho(A)$ :

$$
B^{*}\left(\bar{\beta}-A^{*}\right)^{-1}(\bar{\beta}+\gamma) P(\gamma-A)^{-1} B+\mathbf{G}(\beta)^{*} J \mathbf{G}(\gamma)=R \quad \text { on } U .
$$

REMARK 3.5. If the first two equations in (3.4) hold, then by Lemma 3.4, the left-hand side of the indicator equation in (3.4) does not depend on $\alpha \in \rho(A)$. Thus, if $S, P$, and $J$ are given and if the first two equations in (3.4) hold, then the system is $(R, P, J)$-energy preserving, where $R$ is given by the indicator equation in (3.4). See also Remark 3.9 for other formulas expressing $R$.

Remark 3.6. Suppose that $U, X$, and $Y$ are Hilbert spaces, $A$ is a semigroup generator on $X, C \in \mathcal{L}\left(X_{1} ; Y\right), P=P^{*} \in \mathcal{L}(X)$ is invertible, $J=J^{*} \in \mathcal{L}(Y)$, the first equation in (3.4) holds, and there exists a number $\alpha \in \rho(A)$ such that $-\bar{\alpha} \in \rho(A)$. Then we can construct all the system nodes $S$ with main operator $A$ and observation operator $C$ which are $(R, P, J)$-energy preserving for some $R=R^{*} \in \mathcal{L}(U)$ as follows: Choose $\mathbf{G}(\alpha) \in \mathcal{L}(U ; Y)$ and define $B \in \mathcal{L}\left(U ; X_{-1}\right)$ by

$$
B=-(\alpha-A) P^{-1}\left(\alpha+A^{*}\right)^{-1} C^{*} J \mathbf{G}(\alpha) .
$$

Then the second equation in (3.4) is satisfied and so is the third, if we define $R$ by this equation. By Theorem $3.2, S$ is $(R, P, J)$-energy preserving.

Under a regularity assumption, the previous result can be used to cast the characterization of energy preserving systems into a form that looks more like (1.14).

Definition 3.7. Let $S$ be a system node on $(U, X, Y)$ and let $A, B, C$, and $\mathbf{G}$ be as in Theorem 3.2 ,

(i) $S$ is weakly regular if there exists a $D \in \mathcal{L}(U ; Y)$ such that

$$
\lim _{\alpha \rightarrow+\infty}\left\langle\mathbf{G}(\alpha) u_{0}, y_{0}\right\rangle=\left\langle D u_{0}, y_{0}\right\rangle
$$

for all $u_{0} \in U, y_{0} \in Y$. In this case $D$ is called the feedthrough operator of $S$.

(ii) The weak Yosida extension of $C$ is the operator $C_{w}: \mathcal{D}\left(C_{w}\right) \rightarrow Y$ given by

$$
C_{w} x_{0}:=\underset{\alpha \rightarrow+\infty}{\operatorname{weak}-\lim } \alpha C(\alpha-A)^{-1} x_{0}
$$

with $\mathcal{D}\left(C_{w}\right)$ consisting of those $x_{0} \in X$ for which the above limit exists.

(iii) The weak Yosida extension of $B^{*}$ is the operator $B_{w}^{*}: \mathcal{D}\left(B_{w}^{*}\right) \rightarrow U$ given by

$$
B_{w}^{*} x_{0}:=\underset{\alpha \rightarrow+\infty}{\text { weak- } \lim } \alpha B^{*}\left(\alpha-A^{*}\right)^{-1} x_{0},
$$

with $\mathcal{D}\left(B_{w}^{*}\right)$ consisting of those $x_{0} \in X$ for which the above limit exists.

Obviously, a system node $S$ is weakly regular if and only if its dual node $S^{d}$ is weakly regular. We have $\mathcal{D}(A) \subset \mathcal{D}\left(C_{w}\right)$ and $\mathcal{D}\left(A^{*}\right) \subset \mathcal{D}\left(B_{w}^{*}\right)$; see the arguments in [31. Moreover, if $S$ is weakly regular, then for all $\alpha \in \rho(A)$,

$$
\mathbf{G}(\alpha)=C_{w}(\alpha-A)^{-1} B+D, \quad \mathbf{G}(\alpha)^{*}=B_{w}^{*}\left(\bar{\alpha}-A^{*}\right)^{-1} C^{*}+D^{*},
$$

again by some arguments in 31. In particular, $(\alpha-A)^{-1} B U \subset \mathcal{D}\left(C_{w}\right)$ and $\left(\bar{\alpha}-A^{*}\right)^{-1} C^{*} Y \subset \mathcal{D}\left(B_{w}^{*}\right)$. Thus, weakly regular system nodes are compatible (as defined at the end of Section 2), and we can take $\bar{C}$ to be the restriction of $C_{w}$ to $Z$. With this choice of $\bar{C}$, the operator $D$ from (2.17) is the feedthrough operator of $S$. 
TheOREM 3.8. Let $S=\left[\begin{array}{c}A \& B \\ C \& D\end{array}\right]$ be a weakly regular system node on $(U, X, Y)$ with semigroup generator $A$, control operator $B$, observation operator $C$, and feedthrough operator $D$, and let $B_{w}^{*}$ be the weak Yosida extensions of $B^{*}$. Let $R=R^{*} \in \mathcal{L}(U)$, $P=P^{*} \in \mathcal{L}(X)$, and $J=J^{*} \in \mathcal{L}(Y)$. Then $S$ is an $(R, P, J)$-energy preserving if and only if $X_{1} \subset \mathcal{D}\left(B_{w}^{*} P\right),(\gamma-A)^{-1} B U \subset \mathcal{D}\left(B_{w}^{*} P\right)$ for all $\gamma \in \rho(A)$, and

$$
\begin{cases}P A+A^{*} P=-C^{*} J C & \text { on } X_{1}, \\ B_{w}^{*} P+D^{*} J C=0 & \text { on } X_{1}, \\ \underset{\gamma \rightarrow+\infty}{\text { weak- } \lim _{w} B_{w}^{*} P(\gamma-A)^{-1} B+D^{*} J D=R .} & \end{cases}
$$

As a part of the proof of this theorem we show that the condition $X_{1} \subset \mathcal{D}\left(B_{w}^{*} P\right)$ is redundant in the sense that it is implied by the Lyapunov equation in (3.12). Likewise, the condition $(\gamma-A)^{-1} B U \subset \mathcal{D}\left(B_{w}^{*} P\right)$ for all $\gamma \in \rho(A)$ is redundant in the sense that it is implied by the first two equations in (3.12). Furthermore, the Lyapunov equation implies that the restriction of $B_{w}^{*} P$ to $X_{1}$ is continuous, and the first two equations imply that $B_{w}^{*} P(\gamma-A)^{-1} B$ is continuous on $U$. (All of these statements depend on the weak regularity assumption.)

Proof. We show that (3.4) is equivalent to (3.12) under the additional weak regularity assumption. For this, we assume that the Lyapunov equation $P A+A^{*} P=-C^{*} J C$ (which appears in both lists) holds. As in Lemma 3.4, we define

$$
Q=B^{*}\left(\bar{\alpha}-A^{*}\right)^{-1} P(\bar{\alpha}+A)+\mathbf{G}(\alpha)^{*} J C,
$$

and use (3.11) and the Lyapunov equation to write $Q$ in the form

$$
\begin{aligned}
Q & =B_{w}^{*}\left(\bar{\alpha}-A^{*}\right)^{-1}\left(\bar{\alpha} P+P A+C^{*} J C\right)+D^{*} J C \\
& =B_{w}^{*}\left(\bar{\alpha}-A^{*}\right)^{-1}\left(\bar{\alpha}-A^{*}\right) P+D^{*} J C \\
& =B_{w}^{*} P+D^{*} J C \quad \text { on } X_{1} .
\end{aligned}
$$

We conclude that the middle (cross-term) equations in (3.4) and (3.12) are equivalent, whenever the Lyapunov equation holds.

It remains to show that the last (indicator) equations in (3.4) and (3.12) are equivalent to each other whenever the first two equations in (3.4) and (3.12) hold. If the first two equations in (3.4) hold, then by Remark 3.5. $S$ is $(R, P, J)$-energy preserving, where $R$ is given by the indicator equation in (3.4). Thus, the only thing which we have to show is that the indicator equations in (3.4) and (3.12) define the same operator $R$. Define $R$ by (3.10), i.e.,

$$
R=B^{*}\left(\bar{\beta}-A^{*}\right)^{-1}(\bar{\beta}+\gamma) P(\gamma-A)^{-1} B+\mathbf{G}(\beta)^{*} J \mathbf{G}(\gamma) .
$$

By Lemma 3.4, $R$ does not depend on $\beta, \gamma \in \rho(A)$, so that it coincides with the operator $R$ defined by the indicator equation in (3.4). Let $\beta=\bar{\beta} \rightarrow+\infty$. Then the weak limit of the last term above exists, so the weak limit of the first term on the right-hand side also exists. This implies that, for all $u_{0} \in U$ and $\gamma \in \rho(A), P(\gamma-A)^{-1} B u_{0} \in \mathcal{D}\left(B_{w}^{*}\right)$, and that for all $\gamma \in \rho(A)$,

$$
R=B_{w}^{*} P(\gamma-A)^{-1} B+D^{*} J \mathbf{G}(\gamma) .
$$

Taking the weak limit as $\gamma \rightarrow \infty$ we get the indicator equation in (3.12). 
Note that if $B \in \mathcal{L}(U ; X)$, then the indicator equation in (3.12) reduces to $D^{*} J D=R$, and we recover the equations (1.14).

REMARK 3.9. In the weakly regular case, the indicator equation in (3.12) can be written in still another equivalent form, namely,

$$
R=D^{*} J D+\left(B_{w}^{*} P+D^{*} J C_{w}\right)(\gamma-A)^{-1} B
$$

for some (hence, for all) $\gamma \in \rho(A)$. To see this, it suffices to substitute (3.11) into (3.13). In the strongly regular case (i.e., when $\lim _{\alpha \rightarrow+\infty} \mathbf{G}(\alpha) u_{0}$ exists in $Y$ for each $u_{0} \in U$ ) it is possible to let $\alpha=\bar{\alpha} \rightarrow+\infty$ in the last equation in (3.4) to get one more equivalent form of this equation:

$$
R=D^{*} J D+2 \lim _{\alpha \rightarrow+\infty} \alpha B^{*}\left(\alpha-A^{*}\right)^{-1} P(\alpha-A)^{-1} B .
$$

4. Conservative systems. In the last section the weighting operators $R, P$, and $J$ were only assumed to be self-adjoint. In this section we also require them to have bounded inverses. This enables us to talk about $(R, P, J)$-conservative systems.

Definition 4.1. Let $U, X$, and $Y$ be Hilbert spaces, and let $R=R^{*} \in \mathcal{L}(U), P=$ $P^{*} \in \mathcal{L}(X)$, and $J=J^{*} \in \mathcal{L}(Y)$ be invertible. A system node $S$ is $(R, P, J)$-conservative if it is $(R, P, J)$-energy preserving and $S^{*}$ is $\left(J^{-1}, P^{-1}, R^{-1}\right)$-energy preserving. An $(R, P, J)$-conservative system node is $(R, P, J)$-tory if its control operator is one-to-one and its observation operator has dense range. Instead of " $(I, I, I)$-conservative" we write "conservative" and similarly for "tory".

In many applications it is known a priori that the control operator is one-to-one and that the observation operator has dense range. A conservative system is tory if and only if its transfer function is purely contractive; see Theorem 6.2.

As shown in [3], every contractive analytic (Schur) function in $\mathbb{C}_{+}$is the transfer function of a simple conservative system $S$. A conservative system is called simple if it has no nontrivial subspace which is both uncontrollable and unobservable, or equivalently, if its semigroup $\mathbb{T}$ is completely nonunitary. All simple conservative realizations of a transfer function are equivalent up to a unitary similarity transformation in the state space 2 For more details, see [3] or [24, Chapter 11] for the continuous-time case, and [8] or [27, pp. 255-256] for the discrete-time case.

We proceed to give equivalent conditions for a system node $S$ to be $(R, P, J)$-conservative. Some such conditions are obtained from Theorem 3.2. applied to both $S$ and to the adjoint node $S^{*}$. However, we can replace some of the resulting conditions by others that may be more practical to work with in applications.

Since isometric operators with dense range are unitary (also in the indefinite case), we get the following:

Theorem 4.2. Let $R=R^{*} \in \mathcal{L}(U), P=P^{*} \in \mathcal{L}(X)$, and $J=J^{*} \in \mathcal{L}(Y)$ have bounded inverses. Let $S=\left[\begin{array}{c}A \& B \\ C \& D\end{array}\right]$ be an $(R, P, J)$-energy preserving system node on $(U, X, Y)$ with domain $V$, main operator $A$, control operator $B$, observation operator $C$,

\footnotetext{
${ }^{2}$ A simple conservative system is generally not minimal (in the usual system theoretic sense). Indeed, all minimal finite-dimensional conservative systems have inner transfer functions, so every conservative realization of a transfer function which is not inner must be infinite-dimensional.
} 
and transfer function $\mathbf{G}$, and let $S^{*}=\left[\begin{array}{c}{[A \& B]^{d}} \\ {[C \& D]^{d}}\end{array}\right]$ be the adjoint system node with domain $V^{d}$. Then the following statements are equivalent:

(i) $S$ is $(R, P, J)$-conservative;

(ii) the operator $\left[\begin{array}{ll}P & 0 \\ 0 & J\end{array}\right]\left[\begin{array}{cc}I & 0 \\ C \& D & D\end{array}\right]$ maps $V$ onto $V^{d}$;

(iii) for some (hence, for all) $\alpha \in \mathbb{C}_{+} \cap \rho(A)$, the operator $\left[\begin{array}{ll}\mathbf{A}(\alpha) & \mathbf{B}(\alpha) \\ \mathbf{C}(\alpha) & \mathbf{D}(\alpha)\end{array}\right]$ defined in $\left(\begin{array}{ll}3.5 \\ )\end{array}\right)$ is $\left(\left[\begin{array}{ll}P & 0 \\ 0 & R\end{array}\right],\left[\begin{array}{ll}P & 0 \\ 0 & J\end{array}\right]\right)$-unitary;

(iv) $\left[\begin{array}{c}A \& B \\ C \& D\end{array}\right]+\left[\begin{array}{cc}\bar{\alpha} & 0 \\ 0 & 0\end{array}\right]$ has dense range for some (hence, for all) $\alpha \in \mathbb{C}_{+} \cap \rho(A)$;

(v) $\overline{\operatorname{Ran} C \& D}=Y$, and $P^{-1} A^{*}+A P^{-1}=-B R^{-1} B^{*}$ on $X_{1}^{d}$.

When these equivalent conditions hold, then $\operatorname{Ran} C \& D=Y$, and $\left[\begin{array}{c}A \& B \\ C \& D\end{array}\right]+\left[\begin{array}{cc}\bar{\alpha} & 0 \\ 0 & 0\end{array}\right]$ has a bounded inverse for every $\alpha \in \mathbb{C}_{+} \cap \rho(A)$.

Proof. (i) $\Rightarrow$ (ii): Suppose (i). By point (iii) of Theorem 3.2, (ii) holds if we replace the word "onto" by the word "into". To verify that $\left[\begin{array}{ll}P & 0 \\ 0 & J\end{array}\right]\left[\begin{array}{cc}I & 0 \\ C \& & D\end{array}\right]$ maps $V$ onto $V^{d}$, we apply statement (iii) of Theorem 3.2 to $S^{*}$, and obtain

$$
C \& D\left[\begin{array}{cc}
P^{-1} & 0 \\
0 & R^{-1}
\end{array}\right]\left[\begin{array}{cc}
I & 0 \\
{[C \& D]^{d}}
\end{array}\right]=\left[\begin{array}{ll}
0 & J^{-1}
\end{array}\right] \quad \text { on } V^{d} .
$$

Therefore

$$
\left[\begin{array}{ll}
P & 0 \\
0 & J
\end{array}\right]\left[\begin{array}{cc}
I & 0 \\
C \& D
\end{array}\right]\left[\begin{array}{cc}
P^{-1} & 0 \\
0 & R^{-1}
\end{array}\right]\left[\begin{array}{cc}
I & 0 \\
{[C \& D]^{d}}
\end{array}\right]=\left[\begin{array}{ll}
I & 0 \\
0 & I
\end{array}\right] \quad \text { on } V^{d}
$$

where $\left[\begin{array}{cc}P^{-1} & 0 \\ 0 & R^{-1}\end{array}\right]\left[\begin{array}{cc}I & 0 \\ {[C \& D]^{d}}\end{array}\right]$ maps $V^{d}$ into $V$. Hence, $\left[\begin{array}{cc}P & 0 \\ 0 & J\end{array}\right]\left[\begin{array}{cc}I & 0 \\ C \& D\end{array}\right]$ maps $V$ onto $V^{d}$.

(ii) $\Rightarrow$ (i): If (ii) holds, then arguing as above with $S^{*}$ replaced by $S$, we get

$$
\left[\begin{array}{cc}
P^{-1} & 0 \\
0 & R^{-1}
\end{array}\right]\left[\begin{array}{cc}
I & 0 \\
{[C \& D]^{d}}
\end{array}\right]\left[\begin{array}{ll}
P & 0 \\
0 & J
\end{array}\right]\left[\begin{array}{cc}
I & 0 \\
C \& D
\end{array}\right]=\left[\begin{array}{ll}
I & 0 \\
0 & I
\end{array}\right]
$$

on $V$, i.e., $\left[\begin{array}{ll}P & 0 \\ 0 & J\end{array}\right]\left[\begin{array}{cc}I & 0 \\ C \& D & D\end{array}\right]$ is left-invertible as an operator from $V$ to $V^{d}$. Since it is also onto, it is invertible and $\left(\left[\begin{array}{ll}P & 0 \\ 0\end{array}\right]\left[\begin{array}{cc}I & 0 \\ C \& D\end{array}\right]\right)^{-1}=\left[\begin{array}{cc}P^{-1} & 0 \\ 0 & R^{-1}\end{array}\right]\left[\begin{array}{cc}I & 0 \\ {[C \& D]^{d}}\end{array}\right]$ and (4.1) holds. Multiplying (3.3) by $\left[\begin{array}{cc}P^{-1} & 0 \\ 0 & R^{-1}\end{array}\right]\left[\begin{array}{cc}I & 0 \\ {[C \& D]^{d}}\end{array}\right]$ from the right we find that

$$
[A \& B]^{d}=-P[A \& B]\left[\begin{array}{cc}
P^{-1} & 0 \\
0 & R^{-1}
\end{array}\right]\left[\begin{array}{cc}
I & 0 \\
{[C \& D]^{d}}
\end{array}\right] .
$$

This combined with (4.1) gives

$$
\left[\begin{array}{l}
A \& B \\
C \& D
\end{array}\right]\left[\begin{array}{cc}
P^{-1} & 0 \\
0 & R^{-1}
\end{array}\right]\left[\begin{array}{cc}
I & 0 \\
{[C \& D]^{d}}
\end{array}\right]=\left[\begin{array}{cc}
P^{-1} & 0 \\
0 & J^{-1}
\end{array}\right]\left[\begin{array}{cc}
-[A \& B]^{d} \\
0 & I
\end{array}\right]
$$

Thus, the dual version of (3.3) holds, and so $S$ is conservative.

(i) $\Leftrightarrow$ (iii): This follows from Theorem 3.2, and the fact that when we replace $S$ and $\alpha$ by $S^{*}$ and $\bar{\alpha}$, then the operator $\left[\begin{array}{ll}\mathbf{A}(\alpha) & \mathbf{B}(\alpha) \\ \mathbf{C}(\alpha) & \mathbf{D}(\alpha)\end{array}\right]$ in $(3.5)$ is replaced by $\left[\begin{array}{lll}\mathbf{A}(\alpha) & \mathbf{B}(\alpha) \\ \mathbf{C}(\alpha) & \mathbf{D}(\alpha)\end{array}\right]^{*}$. 
(iii) $\Leftrightarrow$ (iv): It follows from (2.8) that for all $\alpha \in \mathbb{C}_{+} \cap \rho(A)$,

$$
\begin{aligned}
\left(\left[\begin{array}{l}
A \& B \\
C \& D
\end{array}\right]+\left[\begin{array}{cc}
\bar{\alpha} & 0 \\
0 & 0
\end{array}\right]\right) & {\left[\begin{array}{cc}
(\alpha-A)^{-1} & (\alpha-A)^{-1} B \\
0 & I
\end{array}\right] } \\
& =\left[\begin{array}{cc}
\sqrt{2 \Re \alpha} & 0 \\
0 & I
\end{array}\right]\left[\begin{array}{ll}
\mathbf{A}(\alpha) & \mathbf{B}(\alpha) \\
\mathbf{C}(\alpha) & \mathbf{D}(\alpha)
\end{array}\right]\left[\begin{array}{cc}
\frac{1}{\sqrt{2 \Re \alpha}} & 0 \\
0 & I
\end{array}\right],
\end{aligned}
$$

where $\left[\begin{array}{c}(\alpha-A)^{-1} \\ 0\end{array}\left(\begin{array}{c}\alpha-A)^{-1} B \\ I\end{array}\right]\right.$ maps $\left[\begin{array}{l}X \\ U\end{array}\right]$ onto $V$. Therefore $\left[\begin{array}{c}A \& B \\ C \& D\end{array}\right]+\left[\begin{array}{cc}\bar{\alpha} & 0 \\ 0 & 0\end{array}\right]$ has the same range as $\left[\begin{array}{ll}\mathbf{A}(\alpha) & \mathbf{B}(\alpha) \\ \mathbf{C}(\alpha) & \mathbf{D}(\alpha)\end{array}\right]$. By Theorem 3.2, $\left[\begin{array}{ll}\mathbf{A}(\alpha) & \mathbf{B}(\alpha) \\ \mathbf{C}(\alpha) & \mathbf{D}(\alpha)\end{array}\right]$ is $\left(\left[\begin{array}{ll}P & 0 \\ 0 & R\end{array}\right],\left[\begin{array}{ll}P & 0 \\ 0 & J\end{array}\right]\right)$-isometric. Thus, it is $\left(\left[\begin{array}{ll}P & 0 \\ 0 & R\end{array}\right],\left[\begin{array}{ll}P & 0 \\ 0 & J\end{array}\right]\right)$-unitary if and only if $\left(\left[\begin{array}{c}A \& B \\ C \& D\end{array}\right]+\left[\begin{array}{cc}\bar{\alpha} & 0 \\ 0 & 0\end{array}\right]\right)$ has dense range.

(i) $\&($ iv $) \Rightarrow(\mathrm{v})$ : The control Lyapunov equation follows from (i) and Theorem 3.2 . That $C \& D$ has dense range follows from (iv).

(v) $\Rightarrow$ (iii): Assume (v). By Theorem 3.2 .

$$
\left[\begin{array}{cc}
P^{-1} & 0 \\
0 & R^{-1}
\end{array}\right]\left[\begin{array}{cc}
\mathbf{A}(\alpha) & \mathbf{B}(\alpha) \\
\mathbf{C}(\alpha) & \mathbf{D}(\alpha)
\end{array}\right]^{*}\left[\begin{array}{ll}
P & 0 \\
0 & J
\end{array}\right]\left[\begin{array}{cc}
\mathbf{A}(\alpha) & \mathbf{B}(\alpha) \\
\mathbf{C}(\alpha) & \mathbf{D}(\alpha)
\end{array}\right]=\left[\begin{array}{ll}
I & 0 \\
0 & I
\end{array}\right]
$$

This implies that the operator

$$
Q=\left[\begin{array}{ll}
P & 0 \\
0 & J
\end{array}\right]\left[\begin{array}{ll}
\mathbf{A}(\alpha) & \mathbf{B}(\alpha) \\
\mathbf{C}(\alpha) & \mathbf{D}(\alpha)
\end{array}\right]\left[\begin{array}{cc}
P^{-1} & 0 \\
0 & R^{-1}
\end{array}\right]\left[\begin{array}{ll}
\mathbf{A}(\alpha) & \mathbf{B}(\alpha) \\
\mathbf{C}(\alpha) & \mathbf{D}(\alpha)
\end{array}\right]^{*}
$$

is a $\left[\begin{array}{cc}P^{-1} & 0 \\ 0 & J^{-1}\end{array}\right]$-self-adjoint projection on $\left[\begin{array}{l}X \\ Y\end{array}\right]$. By Remark 3.3 applied to $S^{*}$, we have $Q_{11}=I$ ( since we assume the control Lyapunov equation to hold). This combined with the fact that $Q^{2}=Q$ (since $Q$ is a projection) implies that

$$
Q_{12}\left[\begin{array}{ll}
Q_{21} & Q_{22}
\end{array}\right]=0, \quad Q_{22} Q_{21}=0, \quad Q_{21} Q_{12}+Q_{22}^{2}=Q_{22} .
$$

We claim that $Q_{12}=0$. To show this it suffices to show that $\left[\begin{array}{ll}Q_{21} & Q_{22}\end{array}\right]$ has dense range (see the first equation above). By (4.3), the operator $\left[\begin{array}{ll}\mathbf{A}(\alpha) & \mathbf{B}(\alpha) \\ \mathbf{C}(\alpha) & \mathbf{D}(\alpha)\end{array}\right]^{*}$ is onto, and therefore, by (4.4), $\left[\begin{array}{ll}Q_{21} & Q_{22}\end{array}\right]$ has dense range if and only if $\left[\begin{array}{lll}\mathbf{C}(\alpha) & \mathbf{D}(\alpha)\end{array}\right]$ has dense

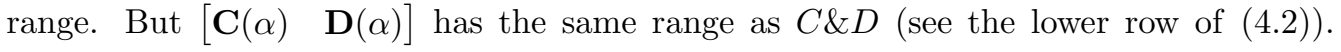
Thus, we conclude that $\left[\begin{array}{ll}Q_{21} & Q_{22}\end{array}\right]$ has dense range and that $Q_{12}=0$. That $Q$ is $\left[\begin{array}{cc}P^{-1} & 0 \\ 0 & J^{-1}\end{array}\right]$-self-adjoint means explicitly that

$$
\left[\begin{array}{cc}
P^{-1} & 0 \\
0 & R^{-1}
\end{array}\right]\left[\begin{array}{ll}
Q_{11} & Q_{12} \\
Q_{21} & Q_{22}
\end{array}\right]=\left[\begin{array}{ll}
Q_{11}^{*} & Q_{21}^{*} \\
Q_{12}^{*} & Q_{22}^{*}
\end{array}\right]\left[\begin{array}{cc}
P^{-1} & 0 \\
0 & R^{-1}
\end{array}\right],
$$

and therefore $Q_{21}=R Q_{12}^{*} P^{-1}=0$. Substituting $Q_{12}=0$ and $Q_{21}=0$ into (4.5) we find that $Q_{22}$ is a projection. We observed earlier that the range of $\left[\begin{array}{ll}Q_{21} & Q_{22}\end{array}\right]$ is dense, and since $Q_{21}=0$, this means that $Q_{22}$ has dense range. Thus $Q_{22}=I$, and the left-hand side of (4.4) is the identity.

Having shown that (i) $-(\mathrm{v})$ are equivalent, we proceed to prove the additional claims.

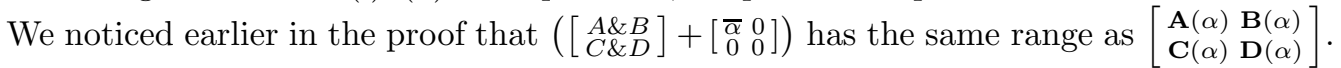
By (iii) this is all of $\left[\begin{array}{c}X \\ Y\end{array}\right]$. In particular, $C \& D$ maps $V$ onto $Y$. That $\left(\left[\begin{array}{c}A \& B \\ C \& D\end{array}\right]+\left[\begin{array}{cc}\bar{\alpha} & 0 \\ 0 & 0\end{array}\right]\right)$ has a bounded inverse for all $\alpha \in \mathbb{C}_{+} \cap \rho(A)$ follows from (4.2). 
REMARK 4.3. By equations (3.3) and Theorem 4.2(ii), each $(R, P, J)$-conservative systems node $S$ satisfies

$$
S^{\dagger}:=\left[\begin{array}{cc}
P^{-1} & 0 \\
0 & R^{-1}
\end{array}\right] S^{*}\left[\begin{array}{cc}
P & 0 \\
0 & J
\end{array}\right]=S^{\leftarrow}:=\left[\begin{array}{cc}
-[A \& B \\
0 & 1
\end{array}\right]\left[\begin{array}{cc}
I & 0 \\
C \& D
\end{array}\right]^{-1}
$$

on $V^{d}$. As $S^{*}$ is a system node, it follows easily that $S^{\dagger}$ is a system node, too. We call $S^{\leftarrow}$ the time-flow-inverse of $S$. The reason for this is the following (see [24, Section 6.5], or in the well-posed case [26, for details): Suppose that $u \in C([0, \tau] ; U), x \in C^{1}([0, \tau] ; X)$, and $y \in C([0, \tau] ; Y)$ satisfy (2.12) on some time interval $[0, \tau]$. Define $\tilde{u}(t)=u(\tau-t)$, $\tilde{x}(t)=x(\tau-t)$, and $\tilde{y}(t)=y(\tau-t)$ for $t \in[0, \tau]$. Then

$$
\left[\begin{array}{c}
\dot{\tilde{x}}(t) \\
\tilde{u}(t)
\end{array}\right]=S^{\leftarrow}\left[\begin{array}{c}
\tilde{x}(t) \\
\tilde{y}(t)
\end{array}\right], \quad t \in[0, \tau] .
$$

In other words, to get from $S$ to $S \leftarrow$ we interchange $u$ and $y$, and at the same time change the direction of time.

Let us proceed to characterize tory systems.

TheOREm 4.4. Let $S=\left[\begin{array}{c}A \& B \\ C \& D\end{array}\right]$ be a system node with main operator $A$, control operator $B$ with Ker $B=\{0\}$, and observation operator $C$ with $\operatorname{Ker} C^{*}=\{0\}$. Let $R=R^{*} \in$ $\mathcal{L}(U), P=P^{*} \in \mathcal{L}(X)$, and $J=J^{*} \in \mathcal{L}(Y)$ have bounded inverses. Then $S$ is $(R, P, J)$ conservative if and only if the two Lyapunov equations

$$
P A+A^{*} P=-C^{*} J C \quad \text { on } X_{1}, \quad P^{-1} A^{*}+A P^{-1}=-B R^{-1} B^{*} \quad \text { on } X_{1}^{d},
$$

and one of the two cross-term equations

$$
\begin{gathered}
{[C \& D]^{d}\left[\begin{array}{c}
P \\
J C
\end{array}\right]=0 \text { on } X_{1},} \\
C \& D\left[\begin{array}{c}
P^{-1} \\
R^{-1} B^{*}
\end{array}\right]=0 \text { on } X_{1}^{d},
\end{gathered}
$$

hold. If this is the case, then both (4.7) and (4.8) hold, as well as the following two indicator equations (for all $\beta, \gamma \in \rho(A)$ ),

$$
\begin{array}{ll}
B^{*}\left(\bar{\beta}-A^{*}\right)^{-1}(\bar{\beta}+\gamma) P(\gamma-A)^{-1} B+\mathbf{G}(\beta)^{*} J \mathbf{G}(\gamma)=R & \text { on } U, \\
C(\beta-A)^{-1}(\beta+\bar{\gamma}) P^{-1}\left(\bar{\gamma}-A^{*}\right)^{-1} C^{*}+\mathbf{G}(\beta) R^{-1} \mathbf{G}(\gamma)^{*}=J^{-1} & \text { on } Y .
\end{array}
$$

In other words, for tory system nodes satisfying the two Lyapunov equations (4.6), the two cross-term equations (4.7) and (4.8) are equivalent, and the indicator equations are redundant. Note that the equations (4.6) imply that $\left[\begin{array}{c}P \\ J C\end{array}\right] X_{1} \subset V^{d}$ and $\left[\begin{array}{c}P^{-1} \\ R^{-1} B^{*}\end{array}\right] X_{1}^{d} \subset$ $V$, so that (4.7) and (4.8) make sense.

Proof. That (4.6)-(4.8) are necessary conditions for conservativity follows from Theorem 3.2 (applied both to the original node $S$ and the dual node $S^{d}$ ). Furthermore, when we pass for $S$ to $S^{d}$ the two conditions (4.7) and (4.8) change places, so it suffices to show that (4.6) and (4.7) imply conservativity.

Assume that (4.6) and (4.7) hold. Then we know some of the resulting elements when

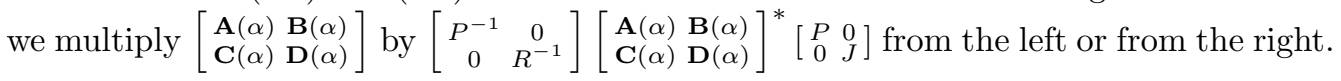


More precisely, these products are of the form

$$
\begin{aligned}
& {\left[\begin{array}{cc}
P^{-1} & 0 \\
0 & R^{-1}
\end{array}\right]\left[\begin{array}{cc}
\mathbf{A}(\alpha) & \mathbf{B}(\alpha) \\
\mathbf{C}(\alpha) & \mathbf{D}(\alpha)
\end{array}\right]^{*}\left[\begin{array}{ll}
P & 0 \\
0 & J
\end{array}\right]\left[\begin{array}{ll}
\mathbf{A}(\alpha) & \mathbf{B}(\alpha) \\
\mathbf{C}(\alpha) & \mathbf{D}(\alpha)
\end{array}\right]=\left[\begin{array}{cc}
I & 0 \\
0 & K
\end{array}\right]} \\
& {\left[\begin{array}{cc}
\mathbf{A}(\alpha) & \mathbf{B}(\alpha) \\
\mathbf{C}(\alpha) & \mathbf{D}(\alpha)
\end{array}\right]\left[\begin{array}{cc}
P^{-1} & 0 \\
0 & R^{-1}
\end{array}\right]\left[\begin{array}{ll}
\mathbf{A}(\alpha) & \mathbf{B}(\alpha) \\
\mathbf{C}(\alpha) & \mathbf{D}(\alpha)
\end{array}\right]^{*}\left[\begin{array}{ll}
P & 0 \\
0 & J
\end{array}\right]=\left[\begin{array}{cc}
I & L J \\
L^{*} P & M
\end{array}\right],}
\end{aligned}
$$

where $K, L$, and $M$ are unknown operators: According to Remark 3.3 the two identity operators in the right-hand sides of (4.9) and (4.10) follow from the two Lyapunov equations in (4.6), and the zero in the right-hand side of (4.9) follows from the cross-term equation in (4.7).

Multiplying the first equation by $\left[\begin{array}{lll}\mathbf{A}(\alpha) & \mathbf{B}(\alpha) \\ \mathbf{C}(\alpha) & \mathbf{D}(\alpha)\end{array}\right]$ from the left and the second equation by the same operator from the right, we get two different expressions which must be equal. Equating the two expressions, we get the identity

$$
\left[\begin{array}{ll}
\mathbf{A}(\alpha) & \mathbf{B}(\alpha) K \\
\mathbf{C}(\alpha) & \mathbf{D}(\alpha) K
\end{array}\right]=\left[\begin{array}{cc}
\mathbf{A}(\alpha)+L J \mathbf{C}(\alpha) & \mathbf{B}(\alpha)+L J \mathbf{D}(\alpha) \\
L^{*} P \mathbf{A}(\alpha)+M \mathbf{C}(\alpha) & L^{*} P \mathbf{B}(\alpha)+M \mathbf{D}(\alpha)
\end{array}\right] .
$$

Hence $L J \mathbf{C}(\alpha)=0$. Since $\mathbf{C}(\alpha)$ has dense range (it has the same range as $C$ ), this implies that $L=0$. Substituting this into (4.11) and using that $\mathbf{C}(\alpha)$ has dense range and $\mathbf{B}(\alpha)$ is one-to-one (since $B$ is one-to-one), we get $K=I$ and $M=I$. This means that $\left[\begin{array}{ll}\mathbf{A}(\alpha) & \mathbf{B}(\alpha) \\ \mathbf{C}(\alpha) & \mathbf{D}(\alpha)\end{array}\right]$ is $\left(\left[\begin{array}{ll}P & 0 \\ 0 & R\end{array}\right],\left[\begin{array}{ll}P & 0 \\ 0 & J\end{array}\right]\right)$-unitary. By Theorem 4.2 $S$ is $(R, P, J)$-conservative.

We can obtain Theorem 1.9 from Theorem 4.4 by taking $R=I, P=I$, and $J=I$, assuming that $S$ is compatible, and writing $[C \& D]\left[\begin{array}{l}x \\ u\end{array}\right]$ in the form $\bar{C} x+D u$. Then (4.6) and (4.8) become the three equations in statement (ii) of Theorem 1.9 .

To the extent described in the following theorem, the non-tory case can be reduced to the tory case. Here we shall require $U_{0}:=\operatorname{Ker} B$ and $Y_{1}:=\overline{\operatorname{Ran} C}$ to be orthocomplemented (or regular) in $U$ respectively $Y$ with respect to the (possibly indefinite) inner product induced by $R$ respectively $J$. For example, in the case of $U_{0}$, this means that $U$ is the direct sum of $U_{0}$ and $U_{1}$, where $U_{1}:=\left(R U_{0}\right)^{\perp}$ is the $R$-orthogonal complement of $U_{0}$. We write this as $U=\left[\begin{array}{l}U_{0} \\ U_{1}\end{array}\right]$. Since $U_{0}$ and $U_{1}$ are closed subspaces of $U$ they inherit the Hilbert space structure of $U$. The additional assumption that they are orthocomplemented implies that they also inherit the Krein space structure of $U$ equipped with the (possibly indefinite) Krein inner product $[\cdot, \cdot]_{U}:=\langle\cdot, R \cdot\rangle_{U}$ (see, e.g., [5, Theorems V.1.3 and V.3.4]). Thus, there exists an invertible self-adjoint Gram operator $R_{0} \in \mathcal{L}\left(U_{0}\right)$ such that the Krein space inner product in $U_{0}$ is given by $[u, v]_{U_{0}}=\left\langle u, R_{0} v\right\rangle_{U_{0}}$, where $[u, v]_{U_{0}}=[u, v]_{U}$ and $\langle u, v\rangle_{U_{0}}=\langle u, v\rangle_{U}$ for all $u, v \in U_{0}$. Clearly, $R_{0}$ is given by $R_{0}=\pi R_{\mid U_{0}}$, where $\pi$ is the orthogonal (with respect to the standard inner product) projection in $U$ whose range is $U_{0}$. The assumptions that $U_{0}$ and $Y_{1}$ are orthocomplemented are far from trivial, unless $R>0$ respectively $J>0$.

Theorem 4.5. Let $S=\left[\begin{array}{c}A \& B \\ C \& D\end{array}\right]$ be a system node on $(U, X, Y)$ with transfer function $\mathbf{G}$. Let $S^{*}=\left[\begin{array}{l}{[A \& B]^{d}} \\ {[C \& D]^{d}}\end{array}\right]$ be the adjoint of $S$. Let $R=R^{*} \in \mathcal{L}(U), P=P^{*} \in \mathcal{L}(X)$, and $J=$ $J^{*} \in \mathcal{L}(Y)$ have bounded inverses. Suppose that $U_{0}:=\operatorname{Ker} B$ is $R$-orthocomplemented in $U$ and that $Y_{1}:=\overline{\operatorname{Ran} C}$ is a $J$-orthocomplemented in $Y$ (see the discussion above). Split $U$ and $Y$ into $U=\left[\begin{array}{l}U_{1} \\ U_{0}\end{array}\right]$ and $Y=\left[\begin{array}{l}Y_{1} \\ Y_{0}\end{array}\right]$, where $U_{1}:=\left(R U_{0}\right)^{\perp}$ and $Y_{0}=\left(J Y_{1}\right)^{\perp}$, 
and let $R_{0}, R_{1}, J_{0}$, and $J_{1}$ be the Gram operators of $U_{0}, U_{1}, Y_{0}$, respectively $Y_{1}$. Finally, decompose $S$ and $\mathbf{G}$ accordingly into

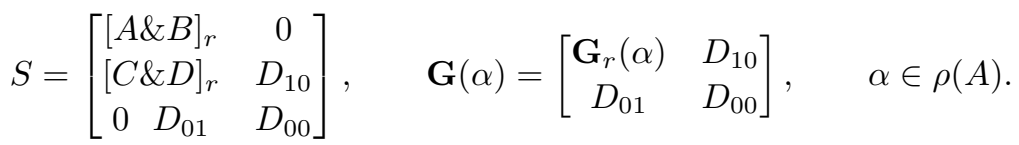

Then the following conditions are equivalent:

(i) $S$ is $(R, P, J)$-conservative;

(ii) $D_{00}$ is $\left(R_{0}, J_{0}\right)$-unitary, $D_{10}=0, D_{01}=0$, and $S_{r}=\left[\begin{array}{c}{[A \& B]_{r}} \\ {[C \& D]_{r}}\end{array}\right]$ is an $\left(R_{1}, P, J_{1}\right)$ tory node;

(iii) $D_{00}$ is $\left(R_{0}, J_{0}\right)$-unitary, $D_{01}=0$, and the two Lyapunov equations (4.6) and the cross-term equation (4.7) hold;

(iv) $D_{00}$ is $\left(R_{0}, J_{0}\right)$-unitary, and the two Lyapunov equations (4.6) and both crossterm equations (4.7) and (4.8) hold.

When these conditions hold, then $\mathbf{G}(\alpha)$ maps $U_{0}\left(R_{0}, J_{0}\right)$-unitarily onto $Y_{0}$ for each $\alpha \in \rho(A)$. In particular, $\operatorname{dim} \operatorname{Ker} B=\operatorname{codim} \operatorname{Ran} C$.

REMARK 4.6. In the following proof we shall use the Krein space adjoints of various operators rather than the ordinary Hilbert space adjoints. We use the superscript $\dagger$ to denote a Krein space adjoint. These adjoints can easily be expressed in terms of the ordinary Hilbert space adjoints and the corresponding weight (Gram) operators, as we did in Section 1 (after Theorem 1.9) for the finite-dimensional case. Thus,

$$
A^{\dagger}=P^{-1} A^{*} P, \quad B^{\dagger}=R^{-1} B^{*} P, \quad C^{\dagger}=P^{-1} C^{*} J, \quad \mathbf{G}(\alpha)^{\dagger}=R^{-1} \mathbf{G}(\alpha)^{*} J,
$$

and

$$
\begin{gathered}
{\left[\begin{array}{l}
A \& B \\
C \& D
\end{array}\right]^{\dagger}=\left[\begin{array}{cc}
P^{-1} & 0 \\
0 & R^{-1}
\end{array}\right]\left[\begin{array}{l}
A \& B \\
C \& D
\end{array}\right]^{*}\left[\begin{array}{cc}
P & 0 \\
0 & J
\end{array}\right],} \\
{\left[\begin{array}{ll}
\mathbf{A}(\alpha) & \mathbf{B}(\alpha) \\
\mathbf{C}(\alpha) & \mathbf{D}(\alpha)
\end{array}\right]^{\dagger}=\left[\begin{array}{cc}
P^{-1} & 0 \\
0 & R^{-1}
\end{array}\right]\left[\begin{array}{ll}
\mathbf{A}(\alpha) & \mathbf{B}(\alpha) \\
\mathbf{C}(\alpha) & \mathbf{D}(\alpha)
\end{array}\right]^{*}\left[\begin{array}{cc}
P & 0 \\
0 & J
\end{array}\right],}
\end{gathered}
$$

where $\left[\begin{array}{ll}\mathbf{A}(\alpha) & \mathbf{B}(\alpha) \\ \mathbf{C}(\alpha) & \mathbf{D}(\alpha)\end{array}\right]$ is the operator defined in $(3.5) 3$ The main advantage of the $\dagger$-adjoints, apart for the notational simplicity, is the following: Since $U_{0}$ and $U_{1}$ are $R$-orthogonal and $Y_{0}$ and $Y_{1}$ are $J$-orthogonal, we can compute $\dagger$-adjoints of various block matrix operators elementwise. Thus, for example, $\mathbf{G}(\alpha)^{\dagger}$ decomposes into $\mathbf{G}(\alpha)^{\dagger}=\left[\begin{array}{cc}\mathbf{G}_{r}(\alpha)^{\dagger} & D_{01}^{\dagger} \\ D_{10}^{\dagger} & D_{00}^{\dagger}\end{array}\right]$, whereas the decomposition of $\mathbf{G}(\alpha)^{*}$ is more complicated.

Proof of Theorem 4.5. We begin with a general observation: The decomposition of $S$ in (4.12) together with (4.2) implies that the operator $\left[\begin{array}{cc}\mathbf{A}(\alpha) & \mathbf{B}(\alpha) \\ \mathbf{C}(\alpha) & \mathbf{D}(\alpha)\end{array}\right] \in \mathcal{L}\left(\left[\begin{array}{l}X \\ U\end{array}\right] ;\left[\begin{array}{l}X \\ Y\end{array}\right]\right)$ defined in (3.5) decomposes into

$$
\left[\begin{array}{ccc}
\mathbf{A}(\alpha) & \mathbf{B}_{r}(\alpha) & 0 \\
\mathbf{C}_{r}(\alpha) & \mathbf{D}_{r}(\alpha) & D_{10} \\
0 & D_{01} & D_{00}
\end{array}\right]:\left[\begin{array}{c}
X \\
U_{1} \\
U_{0}
\end{array}\right] \rightarrow\left[\begin{array}{c}
X \\
Y_{1} \\
Y_{0}
\end{array}\right]
$$

\footnotetext{
${ }^{3}$ The general rule is that one gets the †-adjoint by putting the inverse of the domain space Gram operator to the left and the range space Gram operator to the right of the ordinary adjoint.
} 
Recall that, by Theorem 4.2, $S$ is an $(R, P, J)$-conservative system node if and only if the above operator is unitary with respect to the Krein inner products induced by the weight operators $\left[\begin{array}{ccc}P & 0 & 0 \\ 0 & R_{1} & 0 \\ 0 & 0 & R_{0}\end{array}\right]$ and $\left[\begin{array}{ccc}P & 0 & 0 \\ 0 & J_{1} & 0 \\ 0 & 0 & J_{0}\end{array}\right]$. Using the $\uparrow$-adjoint described in Remark 4.6. this is true if and only if

$$
\left[\begin{array}{ccc}
\mathbf{A}(\alpha)^{\dagger} & \mathbf{C}_{r}(\alpha)^{\dagger} & 0 \\
\mathbf{B}_{r}(\alpha)^{\dagger} & \mathbf{D}_{r}(\alpha)^{\dagger} & D_{01}^{\dagger} \\
0 & D_{10}^{\dagger} & D_{00}^{\dagger}
\end{array}\right]\left[\begin{array}{ccc}
\mathbf{A}(\alpha) & \mathbf{B}_{r}(\alpha) & 0 \\
\mathbf{C}_{r}(\alpha) & \mathbf{D}_{r}(\alpha) & D_{10} \\
0 & D_{01} & D_{00}
\end{array}\right]=\left[\begin{array}{ccc}
I & 0 & 0 \\
0 & I & 0 \\
0 & 0 & I
\end{array}\right]
$$

and

$$
\left[\begin{array}{ccc}
\mathbf{A}(\alpha) & \mathbf{B}_{r}(\alpha) & 0 \\
\mathbf{C}_{r}(\alpha) & \mathbf{D}_{r}(\alpha) & D_{10} \\
0 & D_{01} & D_{00}
\end{array}\right]\left[\begin{array}{ccc}
\mathbf{A}(\alpha)^{\dagger} & \mathbf{C}_{r}(\alpha)^{\dagger} & 0 \\
\mathbf{B}_{r}(\alpha)^{\dagger} & \mathbf{D}_{r}(\alpha)^{\dagger} & D_{01}^{\dagger} \\
0 & D_{10}^{\dagger} & D_{00}^{\dagger}
\end{array}\right]=\left[\begin{array}{ccc}
I & 0 & 0 \\
0 & I & 0 \\
0 & 0 & I
\end{array}\right]
$$

(i) $\Rightarrow$ (ii): If $S$ is energy preserving, then (4.13) holds. The first element in the bottom row of (4.13) gives $D_{10}^{\dagger} \mathbf{C}_{r}(\alpha)=0$. Since $\mathbf{C}_{r}(\alpha)$ has dense range this implies that $D_{10}^{\dagger}=0$, or equivalently, $D_{10}=0$. The last element in the bottom row of (4.13) gives $D_{10}^{\dagger} D_{10}+D_{00}^{\dagger} D_{00}=I$, which simplifies into $D_{00}^{\dagger} D_{00}=I$. Thus $D_{00}$ is $\left(R_{0}, J_{0}\right)$-isometric. By repeating the same argument with (4.13) replaced by (4.14) we conclude that $D_{00}$ is $\left(R_{0}, J_{0}\right)$-unitary, $D_{10}=0$, and $D_{01}=0$. Once we know this much it is easy to show that $\left[\begin{array}{ll}\mathbf{A}(\alpha) & \mathbf{B}_{r}(\alpha) \\ \mathbf{C}_{r}(\alpha) & \mathbf{D}_{r}(\alpha)\end{array}\right]$ must be $\left(\left[\begin{array}{cc}P & 0 \\ 0 & R_{1}\end{array}\right],\left[\begin{array}{ll}P & 0 \\ 0 & J_{1}\end{array}\right]\right)$-unitary, hence $S_{r}$ is $\left(R_{1}, P, J_{1}\right)$-tory.

(ii) $\Rightarrow$ (i): If (ii) holds, then so do (4.13) and (4.14).

(ii) $\Rightarrow$ (iii) and (ii) $\Rightarrow$ (iv) follow from Theorem 3.2 and the fact that (ii) $\Rightarrow$ (i).

(iii) $\Rightarrow$ (ii): Assume (iii). As $B B^{\dagger}=B_{r} B_{r}^{\dagger}$ and $C^{\dagger} C=C_{r}^{\dagger} C_{r}$, it follows that the two Lyapunov equations (4.6) hold with $S$ replaced by $S_{r}$. By Remark 3.3 , the cross-term equation (4.7) implies that $\mathbf{B}(\alpha)^{\dagger} \mathbf{A}(\alpha)+\mathbf{D}(\alpha)^{\dagger} \mathbf{C}(\alpha)=0$. Written in block matrix form this becomes

$$
\left[\begin{array}{c}
\mathbf{B}_{r}(\alpha)^{\dagger} \mathbf{A}(\alpha) \\
0
\end{array}\right]+\left[\begin{array}{c}
\mathbf{D}_{r}(\alpha)^{\dagger} \mathbf{C}_{r}(\alpha) \\
D_{10}^{\dagger} \mathbf{C}_{r}(\alpha)
\end{array}\right]=\left[\begin{array}{l}
0 \\
0
\end{array}\right]
$$

The top row combined with Remark 3.3 implies (4.7) with $S$ replaced by $S_{r}$. By Theorem 4.4. $S_{r}$ is a tory node. The bottom row implies that $D_{10}=0$ (see the paragraph after (4.14)). Thus, (iii) $\Rightarrow$ (ii).

(iv) $\Rightarrow$ (ii): This proof is analogous to the proof of the implication (iii) $\Rightarrow$ (ii) given earlier.

\section{Dissipative systems.}

Definition 5.1. Let $U, X$, and $Y$ be Hilbert spaces, and let $R=R^{*} \in \mathcal{L}(U)$, $P=P^{*} \in \mathcal{L}(X)$, and $J=J^{*} \in \mathcal{L}(Y)$. We call a system node $S$ on $(U, X, Y)(R, P, J)$ dissipative if

$$
\langle x(T), P x(T)\rangle_{X}+\int_{0}^{T}\langle y(t), J y(t)\rangle_{Y} d t \leq\left\langle x_{0}, P x_{0}\right\rangle_{X}+\int_{0}^{T}\langle u(t), R u(t)\rangle_{U} d t,
$$

for all $T>0$ and $x, y, x_{0}$, and $u$ satisfying the conditions of Proposition 2.6. If $R=I$, $P=I$, and $J=I$, then we simply call the node dissipative.

Energy preserving and conservative system nodes are obviously dissipative. 
Proposition 5.2. Let $S=\left[\begin{array}{c}A \& B \\ C \& D\end{array}\right]$ be a system node on $(U, X, Y)$ with domain $V$, main operator $A$, control operator $B$, observation operator $C$, and transfer function $\mathbf{G}$. Let $S^{*}=\left[\begin{array}{l}{[A \& B]^{d}} \\ {[C \& D]^{d}}\end{array}\right]$ be the adjoint system node with domain $V^{d}$, and let $R=R^{*} \in \mathcal{L}(U)$, $P=P^{*} \in \mathcal{L}(X)$, and $J=J^{*} \in \mathcal{L}(Y)$. Then the following statements are equivalent:

(i) $S$ is $(R, P, J)$-dissipative;

(ii) for all $\left[\begin{array}{c}x_{0} \\ u_{0}\end{array}\right] \in V$,

$$
2 \Re\left\langle A \& B\left[\begin{array}{l}
x_{0} \\
u_{0}
\end{array}\right], P x_{0}\right\rangle_{X} \leq\left\langle u_{0}, R u_{0}\right\rangle_{U}-\left\langle C \& D\left[\begin{array}{l}
x_{0} \\
u_{0}
\end{array}\right], J C \& D\left[\begin{array}{l}
x_{0} \\
u_{0}
\end{array}\right]\right\rangle_{Y} ;
$$

(iii) for some (hence, for all) $\alpha \in \rho(A)$, we have

$$
\begin{gathered}
{\left[\begin{array}{cc}
P A+A^{*} P & \left(\alpha+A^{*}\right) P(\alpha-A)^{-1} B \\
B^{*}\left(\bar{\alpha}-A^{*}\right)^{-1} P(\bar{\alpha}+A) & B^{*}\left(\bar{\alpha}-A^{*}\right)^{-1}(2 \Re \alpha) P(\alpha-A)^{-1} B
\end{array}\right]} \\
+\left[\begin{array}{cc}
C^{*} J C & C^{*} J \mathbf{G}(\alpha) \\
\mathbf{G}(\alpha)^{*} J C & \mathbf{G}(\alpha)^{*} J \mathbf{G}(\alpha)
\end{array}\right] \leq\left[\begin{array}{ll}
0 & 0 \\
0 & R
\end{array}\right]
\end{gathered}
$$

which is an operator inequality in $\mathcal{L}\left(\left[\begin{array}{c}X_{1} \\ U\end{array}\right] ;\left[\begin{array}{c}X_{-1}^{d} \\ U\end{array}\right]\right)$;

(iv) for some (hence, for all) $\alpha \in \mathbb{C}_{+} \cap \rho(A)$, the operator $\left[\begin{array}{ll}\mathbf{A}(\alpha) & \mathbf{B}(\alpha) \\ \mathbf{C}(\alpha) & \mathbf{D}(\alpha)\end{array}\right]$ defined in (3.5) is a $\left(\left[\begin{array}{ll}P & 0 \\ 0 & R\end{array}\right],\left[\begin{array}{ll}P & 0 \\ 0 & J\end{array}\right]\right)$-contraction, i.e.,

$$
\left[\begin{array}{ll}
\mathbf{A}(\alpha) & \mathbf{B}(\alpha) \\
\mathbf{C}(\alpha) & \mathbf{D}(\alpha)
\end{array}\right]^{*}\left[\begin{array}{ll}
P & 0 \\
0 & J
\end{array}\right]\left[\begin{array}{ll}
\mathbf{A}(\alpha) & \mathbf{B}(\alpha) \\
\mathbf{C}(\alpha) & \mathbf{D}(\alpha)
\end{array}\right] \leq\left[\begin{array}{ll}
P & 0 \\
0 & R
\end{array}\right]
$$

We leave the proof to the reader. It is almost identical to the proof of Theorem 3.2 , and it is also very similar to the proof of the well-posed version, see [25, Theorem 7.4] (where $R=I, P=I$, and $J=I$ ).

6. The case of positive weight operators. In this final section we take a particular look at the case where the weighting operator $P$ is positive and invertible. Most of the time we shall also assume that $J \geq 0$. We remark that if the system is, in addition, $(R, P, J)$-energy preserving, then it follows from the last (indicator) equation in (3.4) that $R \geq 0$ as well.

As already noticed after Definition 3.1, all $(R, P, J)$-energy preserving (hence, all conservative) system nodes are well posed if both $P$ and $J$ are positive and invertible. The same remark applies to all $(R, P, J)$-dissipative system nodes. It is worth mentioning that when showing that a system node is $(R, P, J)$-dissipative or $(R, P, J)$-energy preserving, well-posedness does not play any role, except as a conclusion if $R$ and $J$ happens to be positive.

In all the main results in Sections 3-4 we have assumed $S$ to be a system node as opposed to an operator node, i.e., we have assumed that $A$ generates a $C_{0}$ semigroup on $X$. If $P$ is positive and invertible and $J \geq 0$, then this assumption can be replaced by the weaker assumption that $\rho(A) \cap \mathbb{C}_{+} \neq 0$.

Theorem 6.1. If $J \geq 0$ and $P$ is positive and invertible, then the conclusions of Theorems 3.2. 4.4, 4.5, and Proposition 5.2 remain valid for any operator node whose main operator $A$ satisfies $\rho(A) \cap \mathbb{C}_{+} \neq \emptyset$. 
Proof. The assumptions of all these results include either the Lyapunov equation $P A+$ $A^{*} P=-C^{*} J C$ or the corresponding inequality $P A+A^{*} P \leq-C^{*} J C$. Since $J \geq 0$, this implies that $P A+A^{*} P \leq 0$. Multiply this identity by $P^{-1 / 2}$ to the left and right to get $P^{1 / 2} A P^{-1 / 2}+P^{-1 / 2} A^{*} P^{1 / 2} \leq 0$. This means that the operator $\tilde{A}=P^{1 / 2} A P^{-1 / 2}$ is dissipative. It is similar to $A$, so it has the same spectrum. Thus, in particular, $\rho(\tilde{A}) \cap C_{+} \neq 0$. This implies that $\tilde{A}$ is maximal dissipative, and by the Lumer-Phillips theorem, it generates a $C_{0}$ contraction semigroup. As $A$ is similar to $\tilde{A}, A$ also generates a $C_{0}$ semigroup (which is a contraction semigroup in the norm induced by $P$.)

A conservative system node with $P>0$ is tory if and only if its transfer function is purely $(R, J)$-contractive:

Theorem 6.2. Let $S$ be an $(R, P, J)$-conservative system node with $P>0$. Then $S$ is tory if and only if $S^{*}$ is tory, if and only if $B$ is one-to-one, if and only if $C$ has dense range, if and only if its transfer function $\mathbf{G}$ is purely $(R, J)$-contractive on $\mathbb{C}_{+}$in the sense that $\langle\mathbf{G}(\alpha) u, J \mathbf{G}(\alpha) u\rangle_{Y}\left\langle\langle u, R u\rangle_{U}\right.$ for all $\alpha \in \mathbb{C}_{+}$and all nonzero $u \in U$.

Proof. By the final remark in Theorem 4.5, $B$ is one-to-one if and only if $C$ has dense range. This implies that $S$ is tory if and only if $S^{*}$ is tory, if and only if $B$ is one-to-one, if and only if $C$ has dense range. It only remains to show that $S$ is tory if and only if $\mathbf{G}$ is purely $(R, J)$-contractive. It is obvious from Theorem 4.5 that $\mathbf{G}(\alpha)=\mathbf{D}(\alpha)$ is not purely contractive if $S$ is not tory (take some nonzero $u \in \operatorname{Ker} B$ and recall that $D_{00}$ is unitary). Conversely, suppose that $S$ is tory. Then, for all nonzero $u \in U$ we have (see the last equation in (3.4) and recall that $P>0$ ):

$$
\langle u, R u\rangle_{U}=\langle\mathbf{B}(\alpha) u, P \mathbf{B}(\alpha) u\rangle_{X}+\langle\mathbf{D}(\alpha) u, J \mathbf{D}(\alpha) u\rangle_{Y}>\langle\mathbf{D}(\alpha) u, J \mathbf{D}(\alpha) u\rangle_{Y}
$$

since $\mathbf{B}(\alpha)$ is one-to-one. Hence $\mathbf{D}(\alpha)=\mathbf{G}(\alpha)$ is purely $(R, J)$-contractive.

We conclude this paper with a slight addition to Theorem 4.5 which is valid when $R$, $P$, and $J$ are positive.

Corollary 6.3. Let $S=\left[\begin{array}{c}A \& B \\ C \& D\end{array}\right]$ be an operator node on $(U, X, Y)$ with main operator $A$ and transfer function G. Let $S^{*}=\left[\begin{array}{l}{[A \& B]^{d}} \\ {[C \& D]^{d}}\end{array}\right]$ be the adjoint of $S$. Suppose that $\rho(A) \cap \mathbb{C}_{+} \neq 0$, and that $R \in \mathcal{L}(U), P \in \mathcal{L}(X)$, and $J \in \mathcal{L}(Y)$ are positive and invertible. Then each of the conditions (i)-(iv) in Theorem 4.5 is equivalent to:

(v) $D_{00}$ is $\left(R_{0}, J_{0}\right)$-unitary, $S_{r}=\left[\begin{array}{l}{[A \& B]_{r}} \\ {[C \& D]_{r}}\end{array}\right]$ is an $\left(R_{1}, P, J_{1}\right)$-tory node, and $\mathbf{G}(\alpha)$ is an $(R, J)$-contraction for some (hence, for all) $\alpha \in \mathbb{C}_{+}$.

Proof. By Theorem 6.1, $S$ is actually a system node (i.e., $A$ generates a $C_{0}$ semigroup) whenever any one of (i)-(iv) hold.

(i) $\Rightarrow(\mathrm{v})$ : By Remark 3.3 and the positivity of $P$,

$$
R=\mathbf{B}(\alpha)^{*} P \mathbf{B}(\alpha)+\mathbf{D}(\alpha)^{*} J \mathbf{D}(\alpha) \leq \mathbf{D}(\alpha)^{*} J \mathbf{D}(\alpha) .
$$

Thus $\mathbf{G}(\alpha)=\mathbf{D}(\alpha)$ is $(R, J)$-contractive. (Note that the positivity of $R$ and $J$ are not needed for this implication.) 
(v) $\Rightarrow$ (ii): Below we shall use the $\dagger$-adjoints described in Remark 4.6. The $(R, J)$ contractivity of $\mathbf{G}(\alpha)$ means that $\mathbf{G}(\alpha)^{\dagger} \mathbf{G}(\alpha) \leq I$, which in block matrix form becomes

$$
\left[\begin{array}{cc}
\mathbf{G}_{r}(\alpha)^{\dagger} & D_{01}^{\dagger} \\
D_{10}^{\dagger} & D_{00}^{\dagger}
\end{array}\right]\left[\begin{array}{cc}
\mathbf{G}_{r}(\alpha) & D_{10} \\
D_{01} & D_{00}
\end{array}\right] \leq\left[\begin{array}{cc}
I & 0 \\
0 & I
\end{array}\right] .
$$

The bottom right corner of this identity gives $D_{10}^{\dagger} D_{10} \leq I-D_{00}^{\dagger} D_{00}=0$ (recall that $D_{00}$ is $\left(R_{0}, J_{0}\right)$-unitary). The assumptions that $R>0$ and $J>0$ imply that the Krein space inner products induced by $R_{0}$ and $J_{1}$ in $U_{0}$ respectively $Y_{1}$ are positive, and therefore $D_{10}^{\dagger} D_{10} \geq 0$. Thus $D_{10}^{\dagger} D_{10}=0$ and $D_{10}=0$. A similar argument which starts with the fact that $D_{00} D_{00}^{\dagger}=I$ and $\mathbf{G}(\alpha) \mathbf{G}(\alpha)^{\dagger} \leq I$ shows that $D_{01}=0$. Thus (v) implies (ii). (Note that the positivity of $P$ is not needed for this implication.)

\section{REFERENCES}

[1] D. Alpay, A. Dijksma, J. Rovnyak, and H. de Snoo. Schur Functions, Operator Colligations, and Reproducing Kernel Hilbert Spaces, volume 96 of Operator Theory: Advances and Applications. Birkhäuser-Verlag, Basel Boston Berlin, 1997.

[2] D. Z. Arov. Passive linear systems and scattering theory. In Dynamical Systems, Control Coding, Computer Vision, volume 25 of Progress in Systems and Control Theory, pages 27-44. BirkhäuserVerlag, Basel, 1999. MR.1684834 (2000c:47134)

[3] D. Z. Arov and M. A. Nudelman. Passive linear stationary dynamical scattering systems with continuous time. Integral Equations Operator Theory, 24:1-45, 1996. MR1366539 (96k:47016)

[4] J. A. Ball. Conservative dynamical systems and nonlinear Livsic-Brodskii nodes. In Nonselfadjoint Operators and Related Topics: Workshop on Operator Theory and its Applications, volume 73 of Operator Theory: Advances and Applications, pages 67-95. Birkhäuser-Verlag, Basel, 1994. MR 1320543 (96c:47108)

[5] J. Bognár. Indefinite inner product spaces, volume 78 of Ergebnisse der Mathematik und ihrer Grenzgebiete. Springer-Verlag, Berlin, 1974. MR0467261(57:7125)

[6] M. S. Brodskiǔ. Triangular and Jordan Representations of Linear Operators, volume 32 of Translations of Mathematical Monographs. American Mathematical Society, Providence, Rhode Island, 1971. MR0322542(48:904)

[7] M. S. Brodskiu. Unitary operator colligations and their characteristic functions. Russian Math. Surveys, 33:4:159-191, 1978. MR0510672 (80e:47010)

[8] V. M. Brodskiı̌. On operator colligations and their characteristic functions. Soviet Math. Dokl., 12:696-700, 1971.

[9] R. F. Curtain and G. Weiss. Well posedness of triples of operators (in the sense of linear systems theory). In Control and Optimization of Distributed Parameter Systems, volume 91 of International Series of Numerical Mathematics, pages 41-59. Birkhäuser-Verlag, Basel, 1989. MR1033051 (91d:93027)

[10] R. F. Curtain and H. Zwart. An Introduction to Infinite-Dimensional Linear Systems Theory. Springer-Verlag, New York, 1995. MR1351248(96i:93001)

[11] J. W. Helton. Systems with infinite-dimensional state space: the Hilbert space approach. Proceedings of the IEEE, 64:145-160, 1976. MR0416694 (54:4764)

[12] P. D. Lax and R. S. Phillips. Scattering Theory. Academic Press, New York, 1967. MR0217440 (36:530)

[13] M. S. Livšic. Operators, Oscillations, Waves (Open Systems), volume 34 of Translations of Mathematical Monographs. American Mathematical Society, Providence, Rhode Island, 1973. MR0347396 $(49: 12116)$

[14] M. S. Livšic and A. A. Yantsevich. Operator Colligations in Hilbert Spaces. John Wiley \& Sons, New York, 1977.

[15] R. Ober and S. Montgomery-Smith. Bilinear transformation of infinite-dimensional state-space systems and balanced realizations of nonrational transfer functions. SIAM J. Control Optim., 28:438465, 1990. MR:1040469(91d:93019) 
[16] D. Salamon. Infinite dimensional linear systems with unbounded control and observation: a functional analytic approach. Trans. Amer. Math. Soc., 300:383-431, 1987. MR0876460 (88d:93024)

[17] D. Salamon. Realization theory in Hilbert space. Math. Systems Theory, 21:147-164, 1989. MR0977021 (89k:93038)

[18] Y. L. Smuljan. Invariant subspaces of semigroups and the Lax-Phillips scheme. Dep. in VINITI, N 8009-1386, Odessa, 49p., 1986.

[19] O. J. Staffans. Coprime factorizations and well-posed linear systems. SIAM J. Control Optim., 36:1268-1292, 1998. MR1618041 (99g:93049)

[20] O. J. Staffans. Admissible factorizations of Hankel operators induce well-posed linear systems. Systems Control Lett., 37:301-307, 1999. MR1753257(2001f:93022)

[21] O. J. Staffans. J-energy preserving well-posed linear systems. Internat. J. Appl. Math. Comput. Sci., 11:1361-1378, 2001. MR.1885509(2002k:93045)

[22] O. J. Staffans. Passive and conservative continuous-time impedance and scattering systems. Part I: Well-posed systems. Math. Control Signals Systems, 15:291-315, 2002. MR1942089 (2003i:93024)

[23] O. J. Staffans. Passive and conservative infinite-dimensional impedance and scattering systems (from a personal point of view). In Mathematical Systems Theory in Biology, Communication, Computation, and Finance, volume 134 of IMA Volumes in Mathematics and its Applications, pages 375-414. Springer-Verlag, New York, 2002. MR2043247 (2004m:93072)

[24] O. J. Staffans. Well-Posed Linear Systems. Cambridge University Press, Cambridge and New York, 2005. MR 2154892

[25] O. J. Staffans and G. Weiss. Transfer functions of regular linear systems. Part II: the system operator and the Lax-Phillips semigroup. Trans. Amer. Math. Soc., 354:3229-3262, 2002. MR1897398 (2003b:93051)

[26] O. J. Staffans and G. Weiss. Transfer functions of regular linear systems. Part III: inversions and duality. Integral Equations Operator Theory, 49:517-558, 2004. MR2091475 (2005g:93067)

[27] B. Sz.-Nagy and C. Foiaş. Harmonic Analysis of Operators on Hilbert Space. North-Holland, Amsterdam, 1970.

[28] E. R. Tsekanovskii and Y. L. Smuljan. The theory of biextensions of operators in rigged Hilbert spaces. Unbounded operator colligations and characteristic functions. Uspehi Mathem. Nauk SSSR, 32:69-124, 1977. MR0463955 (57:3893)

[29] M. Tucsnak and G. Weiss. How to get a conservative well-posed linear system out of thin air. Part II: controllability and stability. SIAM J. Control and Optim., 42:907-935, 2003. MR2002140 (2004f:93075)

[30] G. Weiss. Admissibility of unbounded control operators. SIAM J. Control Optim., 27:527-545, 1989. MR0993285 (90c:93060)

[31] G. Weiss. Transfer functions of regular linear systems. Part I: characterizations of regularity. Trans. Amer. Math. Soc., 342:827-854, 1994. MR.1179402 (94f:93074)

[32] G. Weiss. Optimal control of systems with a unitary semigroup and with colocated control and observation. Systems Control Lett., 48:329-340, 2003. MR2020648(2004i:49054)

[33] G. Weiss, O. J. Staffans, and M. Tucsnak. Well-posed linear systems - a survey with emphasis on conservative systems. Internat. J. Appl. Math. Comput. Sci., 11:7-34, 2001. MR1835146 (2002f:93068)

[34] G. Weiss and M. Tucsnak. How to get a conservative well-posed linear system out of thin air. I. Wellposedness and energy balance. ESAIM Control Optim. Calc. Var., 9:247-274, 2003. MR1966533 (2004d:93069) 